TRANSACTIONS OF THE

AMERICAN MATHEMATICAL SOCIETY

Volume 354, Number 3, Pages 1153-1176

S 0002-9947(01)02912-9

Article electronically published on October 24, 2001

\title{
DISCRETE DECOMPOSITIONS FOR BILINEAR OPERATORS AND ALMOST DIAGONAL CONDITIONS
}

\author{
LOUKAS GRAFAKOS AND RODOLFO H. TORRES
}

\begin{abstract}
Using discrete decomposition techniques, bilinear operators are naturally associated with trilinear tensors. An intrinsic size condition on the entries of such tensors is introduced and is used to prove boundedness for the corresponding bilinear operators on several products of function spaces. This condition should be considered as the direct analogue of an almost diagonal condition for linear operators of Calderón-Zygmund type. Applications include a reduced $T 1$ theorem for bilinear pseudodifferential operators and the extension of an $L^{p}$ multiplier result of Coifman and Meyer to the full range of $H^{p}$ spaces. The results of this article rely on decomposition techniques developed by Frazier and Jawerth and on the vector valued maximal function estimate of Fefferman and Stein.
\end{abstract}

\section{Introduction AND STATEMENTS OF MAIN RESULTS}

Multilinear operators arise naturally within the framework of Calderón-Zygmund theory. The study of such operators using Littlewood-Paley theory and related decomposition techniques goes back to the work of Coifman and Meyer [5] and has been extensively pursued since then with applications to harmonic analysis and partial differential equations (for example see [4, [3], 2], 23]). The recent progress in the study of the bilinear Hilbert transform by Lacey and Thiele [17] has further stimulated the need for a systematic analysis of bilinear operators. By this we mean operators $T(f, g)$ which act on two functions $f$ and $g$ and are linear in both arguments.

An abstract version of the Schwartz kernel theorem gives that any continuous bilinear operator $T$ defined on products of Schwartz functions on $\mathbf{R}^{n}$ and taking values into tempered distributions, $\mathcal{S}\left(\mathbf{R}^{n}\right) \times \mathcal{S}\left(\mathbf{R}^{n}\right) \rightarrow \mathcal{S}^{\prime}\left(\mathbf{R}^{n}\right)$, must be given in the form

$$
T(f, g)(x)=\int_{\mathbf{R}^{n}} \int_{\mathbf{R}^{n}} K(x, y, z) f(y) g(z) d y d z,
$$

where $K(x, y, z)$ is a tempered distribution on $\mathbf{R}^{n} \times \mathbf{R}^{n} \times \mathbf{R}^{n}$ and the integral in (1) is interpreted in the sense of distributions. Alternatively, using the Fourier

Received by the editors May 20, 1999.

1991 Mathematics Subject Classification. Primary 42B25, 42B20, 47G30; Secondary 42C15, 46E35, 35S99.

Key words and phrases. Singular integrals, maximal functions, Littlewood-Paley theory, almost diagonal condition, multilinear operators, wavelets, Triebel-Lizorkin spaces.

Grafakos' research partially supported by the NSF under grant DMS 9623120.

Torres' research partially supported by the NSF under grant DMS 9696267. 
transform

$$
\widehat{f}(\xi)=\int_{\mathbf{R}^{n}} f(x) e^{-i x \cdot \xi} d x
$$

the bilinear operator in (1) can be realized in pseudodifferential form

$$
T(f, g)(x)=\frac{1}{(2 \pi)^{2 n}} \int_{\mathbf{R}^{n}} \int_{\mathbf{R}^{n}} \sigma(x, \xi, \eta) \widehat{f}(\xi) \widehat{g}(\eta) e^{i x \cdot(\xi+\eta)} d \xi d \eta,
$$

where, at least in the distributional sense, the symbol $\sigma(x, \xi, \eta)$ and the kernel $K(x, y, z)$ are related as in

$$
\mathcal{F}_{2 n}^{-1}(\sigma(x, \xi, \eta))(y, z)=K(x, x-y, x-z) .
$$

Here $\mathcal{F}_{2 n}^{-1}$ denotes the inverse Fourier transform in both variables $(\xi, \eta) \in \mathbf{R}^{2 n}$.

It is very useful to consider both realizations of operators in terms of kernels and symbols since operators arise in both forms: sometimes they naturally act in the "time" domain; sometimes they are better understood through their action in the "frequency" domain. In the linear case the corresponding realizations of operators have led to a careful study of the conditions on kernels and/or symbols that produce bounded operators on several function spaces; in particular simultaneous boundedness on all $L^{p}$ spaces, $1<p<\infty$, a typical feature of Calderón-Zygmund theory. To some extent, the culminating results for operators with singular kernels are the $T 1$ and $T b$ theorems, [7] and [8], and their numerous generalizations. Results in terms of the symbols of pseudodifferential operators in the exotic class $S_{1,1}^{0}$ have been considered in [20, [18], 1], [15] and [16], to name a few. In both cases orthogonality arguments play a crucial role. For example, such orthogonality considerations in the original proof of the $T 1$ theorem by David and Journé are contained in CotlarStein's Lemma. For pseudodifferential operators such orthogonality arguments are subsumed in Littlewood-Paley and "phase-space" decompositions of symbols.

An alternative approach in studying boundedness properties of operators is to decompose the functions on which they act, rather than the operators themselves. This is a recurrent theme in harmonic analysis through the use of atoms, molecules, wavelets, and tiles. The main goal of this approach is to understand the behavior of an operator on those basic building blocks. By decomposing the functions on which an operator acts, one discretizes the operator and studies the corresponding matrix as in the finite dimensional case. One can then look at simple, easily verifiable conditions on these matrices, that guarantee boundedness for the associated operators. This general approach has been systematically carried out in the work of Meyer 19] and of Frazier and Jawerth [10. The authors in [10] have precisely quantified sufficient conditions to imply boundedness for the associated operator on all spaces that admit Littlewood-Paley decomposition. Such conditions, see (13) below, only depend on the size of the entries of the matrix associated to an operator, a fact that makes them easily verifiable in numerous applications. The conditions are referred to as almost diagonal [10, because the size of the entries of the matrix decay as fast as the parameters that index them get farther apart. Remarkably, as the size of the almost diagonal estimates together with the use of smooth molecules suffice to analyze general Calderón-Zygmund operators, not only on $L^{p}$ but also on Sobolev and other function spaces in a unified way (see e.g. [21]). In addition, the almost diagonal estimates are extremely simple to check for pseudodifferential operators in various classes (see [13] and the references therein). It is important to point 
out that, since they are so closely tied to Calderón-Zygmund and Littlewood-Paley theory, the almost diagonal estimates are " $p$-independent" for $1<p<\infty$.

In this work, we carry out the approach above in the bilinear setting. We seek a way to discretize bilinear operators and find sufficient conditions on the associated trilinear discrete tensors that imply continuity. We find a bilinear almost diagonal estimate which is general enough to cover the type of multilinear operators studied in [5] and [6], as well as some new examples. The almost diagonal condition we introduce is intrinsically bilinear in the sense that it does not require reducing the operators to linear ones by freezing one function.

We will be working below in $n$-dimensional Euclidean space. Given a bilinear operators $T(f, g)$ one defines an infinite array of scalars, which we will call a tensor, $A=\{a(\lambda m, \nu k, \mu l)\}$ by setting

$$
a(\lambda m, \nu k, \mu l)=\left\langle T\left(\phi_{\nu k}, \phi_{\mu l}\right), \phi_{\lambda m}\right\rangle,
$$

where $\left\{\phi_{\nu k}\right\}$ is a family of wavelets, $\nu, \mu, \lambda$ range over $\mathbf{Z}$, and $k, l, m$ over $\mathbf{Z}^{n}$. Conversely, any tensor $A$ as above gives rise to a bilinear operator $T$ defined by

$$
T(f, g)=\sum_{\mu, l} \sum_{\nu, k} \sum_{\lambda, m} a(\lambda m, \nu k, \mu l)\left\langle f, \phi_{\nu k}\right\rangle\left\langle g, \phi_{\mu l}\right\rangle \phi_{\lambda m} .
$$

For three real numbers, $a, b, c$, such that $a \leq b \leq c$, we denote by $\operatorname{med}(a, b, c)$ the "medium" number $b$. The following theorem gives a sufficient condition for a bilinear operator $T$ to be bounded on products of $L^{p}$ spaces.

Theorem 1. Suppose that the tensor $\{a(\lambda m, \nu k, \mu l)\}$ associated to the bilinear operator $T$ satisfies the almost diagonal estimate

$$
\begin{gathered}
|a(\lambda m, \nu k, \mu l)| \leq \\
\frac{C 2^{-(\max (\mu, \nu, \lambda)-\min (\mu, \nu, \lambda)) \varepsilon} 2^{-\max (\mu, \nu, \lambda) n / 2} 2^{\operatorname{med}(\mu, \nu, \lambda) n / 2} 2^{\min (\mu, \nu, \lambda) n / 2}}{\left(\left(1+2^{\min (\nu, \mu)}\left|2^{-\nu} k-2^{-\mu} l\right|\right)\left(1+2^{\min (\mu, \lambda)}\left|2^{-\mu} l-2^{-\lambda} m\right|\right)\left(1+2^{\min (\lambda, \nu)}\left|2^{-\lambda} m-2^{-\nu} k\right|\right)\right)^{N}}
\end{gathered}
$$

for some $C>0, N>n$, and $\varepsilon>0$. Then the corresponding operator $T$ can be extended to be a bounded operator from $L^{p}\left(\mathbf{R}^{n}\right) \times L^{q}\left(\mathbf{R}^{n}\right)$ into $L^{r}\left(\mathbf{R}^{n}\right)$ when $1 / p+1 / q=1 / r$ and $1<p, q, r<\infty$.

In Section 6, we also obtain versions of Theorem 10 for Hardy spaces, Sobolev spaces, and other Triebel-Lizorkin spaces. Our methods are in the spirit of the program developed in 10 but some new ideas are employed.

The almost diagonal condition has some symmetries which can be translated into properties of the transposes of $T$. Any bilinear operator has two transposes naturally associated with it. The first transpose $T^{* 1}$ of $T$ is the transpose of the linear operator $f \rightarrow T(f, g)$. $T^{* 1}$ is a bilinear operator with kernel $K^{* 1}(x, y, z)=$ $K(y, x, z)$. The second transpose $T^{* 2}$ of $T$ is the transpose of the linear operator $g \rightarrow T(f, g)$; this is a bilinear operator with kernel $K^{* 2}(x, y, z)=K(z, y, x)$. In other words, for all $f, g$, and $h$ in $\mathcal{S}\left(\mathbf{R}^{n}\right)$ we have

$$
\int_{\mathbf{R}^{n}} T(f, g) h d x=\int_{\mathbf{R}^{n}} T^{* 1}(h, g) f d x=\int_{\mathbf{R}^{n}} T^{* 2}(f, h) g d x .
$$

One can easily check that if a bilinear operator $T$ has symbol $\sigma(\xi, \eta)$ (independent of $x)$, then the first transpose $T^{* 1}$ of $T$ is the bilinear operator with symbol $\sigma^{* 1}(\xi, \eta)=$ $\sigma(-(\xi+\eta), \eta)$ while the second transpose $T^{* 2}$ of $T$ is the bilinear operator with symbol $\sigma^{* 2}(\xi, \eta)=\sigma(\xi,-(\xi+\eta))$. At the discrete level, the transposes of the tensor $A=\{a(\lambda m, \nu k, \mu l)\}$ are $A^{* 1}=\left\{a^{* 1}(\lambda m, \nu k, \mu l)\right\}=\{a(\nu k, \lambda m, \mu l)\}$ and 
$A^{* 2}=\left\{a^{* 2}(\lambda m, \nu k, \mu l)\right\}=\{a(\mu l, \nu k, \lambda m)\}$. Note that if $T$ is bounded on the full range of $1<p, q, r<\infty$ with $1 / p+1 / q=1 / r$, then, by duality, so are its transposes. This is in accordance with the fact that the estimates in Theorem 1 are symmetric under a permutation of variables.

As explained before, it is useful to have a simple way to check the almost diagonal conditions. We do this by introducing the notion of bilinear smooth molecules. These are functions associated to pairs of dyadic cubes and have certain localization properties at two different scales. Bilinear operators that map pairs of basic building blocks into bilinear smooth molecules, satisfy the bilinear almost diagonal estimates and, hence, they are bounded. We have

Theorem 2. Let $T$ be a bilinear operator so that for a wavelet family $\left\{\phi_{\mu l}\right\}$, the three families $\left\{\psi_{\mu l, \lambda m}\right\}=\left\{T\left(\phi_{\mu l}, \phi_{\lambda m}\right)\right\},\left\{\psi_{\mu l, \lambda m}^{* 1}\right\}=\left\{T^{* 1}\left(\phi_{\mu l}, \phi_{\lambda m}\right)\right\}$, and $\left\{\psi_{\mu l, \lambda m}^{* 2}\right\}=\left\{T^{* 2}\left(\phi_{\mu l}, \phi_{\lambda m}\right)\right\}$ are families of bilinear smooth molecules. Then $T$ can be extended to be a bounded operator from $L^{p}\left(\mathbf{R}^{n}\right) \times L^{q}\left(\mathbf{R}^{n}\right)$ into $L^{r}\left(\mathbf{R}^{n}\right)$ when $1 / p+1 / q=1 / r$ and $1<p, q, r<\infty$.

This article is organized as follows. We use the notation in [10], 11], and related papers. Most of it is recalled in Section 2 which also contains some other background material. The reader familiar with the subject can move directly to Section 5 where Theorem 1 is proved. One can then return to Section 3 which contains motivation for the bilinear almost diagonal estimates, the notion of bilinear smooth molecules, and three propositions which, combined with Theorem 1, imply Theorem 2 These three propositions are proved in Section 4. In Section 6 we discuss extensions of Theorem 1 to other function spaces. Examples and applications are given in Section 7.

Acknowledgment. The research presented here originated while the authors were visiting the Mathematical Sciences Research Institute, Berkeley, during the program in Harmonic Analysis, Fall 1997. The authors want to express their gratitude to the institute and the organizers of the program for their support and hospitality.

\section{SOME BACKGROUND TOOLS}

We begin by recalling some of the basics of the work in [10. Throughout this section we fix a function $\phi \in \mathcal{S}\left(\mathbf{R}^{n}\right)$ such that $\widehat{\phi}$ is supported in the annulus $\pi / 4 \leq|\xi| \leq \pi$ and such that $|\widehat{\phi}(\xi)|$ is bounded away from zero on a smaller annulus $\pi / 4+\epsilon \leq|\xi| \leq \pi-\epsilon$. Such a function can be chosen to be real-valued, radial and satisfying

$$
\sum_{\nu \in \mathbf{Z}}\left|\widehat{\phi}\left(2^{\nu} \xi\right)\right|^{2}=1, \quad \xi \neq 0 .
$$

For $\nu \in \mathbf{Z}$ and $k \in \mathbf{Z}^{n}$, let $Q_{\nu k}$ be the dyadic cube

$$
Q_{\nu k}=\left\{\left(x_{1}, \ldots, x_{n}\right) \in \mathbf{R}^{n}: k_{i} \leq 2^{\nu} x_{i}<k_{i}+1, i=1, \ldots, n\right\} .
$$

The lower left-corner of $Q_{\nu k}$ will be denoted by $2^{-\nu} k$ and the characteristic function of the cube by $\chi_{Q_{\nu k}}$. Define

$$
\phi_{\nu k}(x)=2^{\nu n / 2} \phi\left(2^{\nu} x-k\right),
$$


so that $\left\|\phi_{\nu k}\right\|_{L^{2}}=\|\phi\|_{L^{2}}$ and $\widehat{\phi}_{\nu k}$ is supported in the annulus $2^{\nu-2} \pi \leq|\xi| \leq 2^{\nu} \pi$. Observe that

$$
\left|\partial^{\gamma} \phi_{\nu k}(x)\right| \leq \frac{C_{\gamma, N} 2^{\nu n / 2} 2^{|\gamma| \nu}}{\left(1+2^{\nu}\left|x-2^{-\nu} k\right|\right)^{N}},
$$

for any multi-index $\gamma$ and positive integer $N$. A family of functions $\left\{m_{\nu k}\right\}$ (not necessarily obtained by translations and dilations) that satisfy the above estimates as well as the cancellation condition

$$
\int m_{\nu k}(x) d x=0
$$

is called a family of smooth molecules for $L^{p}\left(\mathbf{R}^{n}\right), 1<p<\infty$. Observe that the functions $\phi_{\nu k}$ are molecules with vanishing moments of all orders.

The function $\phi$ can be selected to generate a useful almost orthogonal wavelet decomposition of $L^{p}\left(\mathbf{R}^{n}\right)$. Moreover the following holds.

Theorem A (Frazier and Jawerth [10]). Suppose $f \in L^{p}\left(\mathbf{R}^{n}\right)$ for $1<p<\infty$. Then $f$ can be written as

$$
f=\sum_{\nu, k}\left\langle f, \phi_{\nu k}\right\rangle \phi_{\nu k}
$$

where $\nu$ ranges over $\mathbf{Z}, k$ over $\mathbf{Z}^{n}$, the series in (10) converges in $L^{p}$ and

$$
\|f\|_{L^{p}\left(\mathbf{R}^{n}\right)} \approx\left\|\left(\sum_{\nu}\left(\sum_{k}\left|\left\langle f, \phi_{\nu k}\right\rangle\right| 2^{\nu n / 2} \chi_{Q_{\nu k}}\right)^{2}\right)^{1 / 2}\right\|_{L^{p}\left(\mathbf{R}^{n}\right)} .
$$

The notation $\langle f, g\rangle$ stands for the usual action of a distribution $f$ on a test function $g$. Also for two positive expressions $A$ and $B$ we have $A \approx B$ when they have comparable size with constants independent of the parameters involved. The expression on the left in (11) takes the particularly simple and familiar Parseval's form when $p=2$ since the functions

$$
\tilde{\chi}_{Q_{\nu k}}=2^{\nu n / 2} \chi_{Q_{\nu k}}
$$

are $L^{2}$ normalized. The series in (10) looks then like an orthonormal decomposition but in general it is not. It is possible to replace the function $\phi$ by a family of functions with similar properties and obtain a true orthonormal wavelet basis for $L^{2}\left(\mathbf{R}^{n}\right)$ (see e.g. 19]). Nevertheless, the above almost orthogonal decomposition will suffice for our purposes and simplifies the notation. With some abuse of terminology, we will still refer to $\left\{\phi_{\nu k}\right\}$ as a family of wavelets.

Expressions similar to the one on the right hand side of (11) can be used to characterize other function spaces such as the homogeneous Triebel-Lizorkin spaces $\dot{F}_{p}^{\alpha, s}$ in the entire range of indices $0<p<+\infty, 0<s \leq+\infty$, and $\alpha \in \mathbf{R}$ (see Section 6 below for definitions). In addition the wavelet representation for a function in

$$
\mathcal{S}_{0}=\left\{f \in \mathcal{S}: \partial^{\gamma} \hat{f}(0)=0, \text { for all } \gamma\right\},
$$

converges in the topology of $\mathcal{S}$. The space $\mathcal{S}_{0}$ is clearly dense in all $L^{p}$, for $1<p<$ $\infty$.

Next we discuss almost diagonal operators and the role of wavelets in their study. Suppose that $T$ is a linear operator acting on Schwartz functions on $\mathbf{R}^{n}$. Consider 
the matrix of $T$ with respect to a fixed family $\left\{\phi_{\nu k}\right\}$. This is the matrix

$$
a(\mu l, \nu k)=\left\langle T\left(\phi_{\nu k}\right), \phi_{\mu l}\right\rangle,
$$

where $\nu$ and $\mu$ range over $\mathbf{Z}$ and $k, l$ over $\mathbf{Z}^{n}$. Because of the wavelet representation and Theorem A, the boundedness of the operator $T$ on $L^{p}$ is equivalent to the boundedness of the associated matrix on $\dot{f}_{p}^{0,2}$, the space of sequences $\left\{s_{\nu k}\right\}$ with norm given by

$$
\left\|\left\{s_{\nu k}\right\}\right\|_{f_{p}^{0,2}}=\left\|\left(\sum_{\nu}\left(\sum_{k}\left|s_{\nu k}\right| \widetilde{\chi}_{Q_{\nu k}}\right)^{2}\right)^{1 / 2}\right\|_{L^{p}\left(\mathbf{R}^{n}\right)} .
$$

Conversely, any matrix $A=\{a(\mu l, \nu k)\}$ bounded on $\dot{f}_{p}^{0,2}$ gives rise to a bounded operator $T$ on $L^{p}$ defined by

$$
T f=\sum_{\mu, l} \sum_{\nu, k} a(\mu l, \nu k)\left\langle f, \phi_{\nu k}\right\rangle \phi_{\mu l} .
$$

The following theorem gives a sufficient condition for $T$ to be a bounded operator on $L^{p}\left(\mathbf{R}^{n}\right)$.

Theorem B (Frazier and Jawerth [10]). Suppose that the matrix $\{a(\mu l, \nu k)\}$ of the operator $T$ satisfies the almost diagonal estimate

$$
|a(\mu l, \nu k)| \leq \frac{C 2^{-|\mu-\nu| \varepsilon} 2^{-|\mu-\nu| n / 2}}{\left(1+2^{\min (\mu, \nu)}\left|2^{-\nu} k-2^{-\mu} l\right|\right)^{N}},
$$

for some $C>0, N>n$, and $\varepsilon>0$. Then the corresponding operator $T$ can be extended to be bounded from $L^{p}\left(\mathbf{R}^{n}\right)$ into itself for $1<p<\infty$.

The theorem above is very useful when applied to "reduced" operators, such as operators from which certain quantities have been subtracted to make them have cancellation (in the form of $T 1=T^{*} 1=0$ ). The almost diagonal estimates have proved to be useful technical devices in characterizing function spaces in terms of wavelet coefficients, see [10, [11.

A crucial ingredient in the proof of Theorem B and Theorem 1 is the FeffermanStein vector valued maximal function theorem.

Theorem C (Fefferman and Stein [9]). Let $M$ be the usual Hardy-Littlewood maximal operator on $\mathbf{R}^{n}$. Then for any $1<p<\infty$ and $1<s \leq \infty$ there exists a constant $C$ such that for all sequences $\left\{f_{j}\right\}$ of locally integrable functions the following inequality is valid

$$
\left\|\left(\sum_{j} M\left(f_{j}\right)^{s}\right)^{1 / s}\right\|_{L^{p}\left(\mathbf{R}^{n}\right)} \leq C\left\|\left(\sum_{j}\left|f_{j}\right|^{s}\right)^{1 / s}\right\|_{L^{p}\left(\mathbf{R}^{n}\right)} .
$$

We now set up the corresponding notation for bilinear operators. A given bilinear operator will be associated to a tensor instead of a matrix. By this we mean the infinite array of numbers

$$
a(\lambda m, \nu k, \mu l)=\left\langle T\left(\phi_{\nu k}, \phi_{\mu l}\right), \phi_{\lambda m}\right\rangle,
$$

where $\nu, \mu$, and $\lambda$ range over $\mathbf{Z}$ and $k, l$, and $m$ over $\mathbf{Z}^{n}$ and $\left\{\phi_{\nu k}\right\}$ is a fixed almost orthogonal wavelet family. Again, the wavelet representation of functions discretizes the analysis and reduces the study of bilinear operators $T$ on $L^{p} \times L^{q}$ 
to the study of tensors $A=\{a(\lambda m, \nu k, \mu l)\}$ on $\dot{f}_{p}^{0,2} \times \dot{f}_{q}^{0,2}$. Given any such tensor $A$, we define a bilinear operator

$$
T(f, g)=\sum_{\lambda, m} \sum_{\mu, l} \sum_{\nu, k} a(\lambda m, \nu k, \mu l)\left\langle f, \phi_{\nu k}\right\rangle\left\langle g, \phi_{\mu l}\right\rangle \phi_{\lambda m}
$$

whose corresponding tensor is $A$. Using Theorem $\mathrm{A}$, it is easy to see that the $\dot{f}_{p}^{0,2} \times \dot{f}_{q}^{0,2} \rightarrow f_{r}^{0,2}$ boundedness of $A$ is equivalent to the $L^{p}\left(\mathbf{R}^{n}\right) \times L^{q}\left(\mathbf{R}^{n}\right) \rightarrow L^{r}\left(\mathbf{R}^{n}\right)$ boundedness of $T$.

\section{Motivation for the Almost diagonal COnditions}

As mentioned in the introduction, we want to study an operator by understanding its action on simple elementary functions. Wavelets are very useful in this sense since they are "almost eigenvectors" for Calderón-Zygmund operators. In fact, it is not hard to see that such operators map the family of wavelets into functions which still satisfy estimates (8) for some multi-indices $\gamma$. Moreover, for convolution operators (or operators with $T^{*} 1=0$ ), the cancellation condition (9) is also preserved. Thus, Calderón-Zygmund operators "map wavelets into molecules". All this can be quantified in a precise way. The estimates (8) together with the cancellation in wavelets and molecules can be used to show that the matrix associated to the operator is almost diagonal in the sense of Theorem A. The almost diagonal estimate in that result is motivated by two simple propositions that we now recall. For a proof, see for instance [10].

Proposition 1. Suppose that $\psi_{\nu}$ and $\psi_{\mu}$ are functions defined on $\mathbf{R}^{n}$ satisfying for some $x_{\nu}, x_{\mu}$ in $\mathbf{R}^{n}$ and all $M, N>n$

$$
\begin{aligned}
& \left|\psi_{\nu}(x)\right| \leq \frac{C 2^{\nu n / 2}}{\left(1+2^{\nu}\left|x-x_{\nu}\right|\right)^{N}}, \\
& \left|\psi_{\mu}(x)\right| \leq \frac{C 2^{\mu n / 2}}{\left(1+2^{\mu}\left|x-x_{\mu}\right|\right)^{M}},
\end{aligned}
$$

for some constants $C=C(N, M)>0$. Then, for all $N>n$ there exists a constant $C_{N}>0$ such that the following estimate is valid

$$
\int_{\mathbf{R}^{n}}\left|\psi_{\nu}(x)\right|\left|\psi_{\mu}(x)\right| d x \leq \frac{C_{N} 2^{-|\nu-\mu| n / 2}}{\left(1+2^{\min (\mu, \nu)}\left|x_{\nu}-x_{\mu}\right|\right)^{N}} .
$$

A better estimate can be obtained if one of the functions satisfies size estimates for its derivatives while the other function has cancellation.

Proposition 2. Suppose that $\psi_{\nu}$ and $\psi_{\mu}$ are functions defined on $\mathbf{R}^{n}$ satisfying estimates (15) and (16) for some $x_{\nu}, x_{\mu}$ in $\mathbf{R}^{n}$ and all $M, N>n$. Suppose in addition that

$$
\begin{array}{ll}
\int_{\mathbf{R}^{n}} \psi_{\nu}(x) x^{\gamma} d x=0 & \text { for all }|\gamma| \leq L-1, \\
\left|\partial_{x}^{\gamma} \psi_{\mu}(x)\right| \leq C_{M} \frac{2^{\mu|\gamma|} 2^{\mu n / 2}}{\left(1+2^{\nu}\left|x-x_{\mu}\right|\right)^{M}} & \text { for all }|\gamma| \leq L
\end{array}
$$

for some $L$ nonnegative integer. If $\nu \geq \mu$ and $N>M+L$, then for some constant $C_{N, M, L}>0$ the following estimate is valid

$$
\left|\int_{\mathbf{R}^{n}} \psi_{\nu}(x) \psi_{\mu}(x) d x\right| \leq C_{N, M, L} \frac{2^{-(\nu-\mu)(L+n / 2)}}{\left(1+2^{\mu}\left|x_{\nu}-x_{\mu}\right|\right)^{M}} .
$$


We will now discuss how the bilinear almost diagonal condition in Theorem 1 arises. It is important to convince our reader that the main expression in Theorem 1 appears when one considers estimates involving integrals of products of three wavelets. The propositions below give rise to the bilinear almost diagonal estimate and provide the motivation for this work.

Proposition 3. Suppose that $\psi_{\nu}, \psi_{\mu}, \psi_{\lambda}$ are functions defined on $\mathbf{R}^{n}$ satisfying the following estimates for all $x \in \mathbf{R}^{n}$

$$
\begin{aligned}
& \left|\psi_{\nu}(x)\right| \leq C_{N} \frac{2^{\nu n / 2}}{\left(1+2^{\nu}\left|x-x_{\nu}\right|\right)^{N}}, \\
& \left|\psi_{\mu}(x)\right| \leq C_{N} \frac{2^{\mu n / 2}}{\left(1+2^{\mu}\left|x-x_{\mu}\right|\right)^{N}}, \\
& \left|\psi_{\lambda}(x)\right| \leq C_{N} \frac{2^{\lambda n / 2}}{\left(1+2^{\lambda}\left|x-x_{\lambda}\right|\right)^{N}},
\end{aligned}
$$

for some $x_{\nu}, x_{\mu}, x_{\lambda}$ in $\mathbf{R}^{n}$ and for all $N>n$. Then the following estimate is valid

$$
\begin{gathered}
\int_{\mathbf{R}^{n}}\left|\psi_{\nu}(x)\right|\left|\psi_{\mu}(x)\right|\left|\psi_{\lambda}(x)\right| d x \leq \\
\frac{C_{N} 2^{-\max (\mu, \nu, \lambda) n / 2} 2^{\operatorname{med}(\mu, \nu, \lambda) n / 2} 2^{\min (\mu, \nu, \lambda) n / 2}}{\left(\left(1+2^{\min (\nu, \mu)}\left|x_{\nu}-x_{\mu}\right|\right)\left(1+2^{\min (\mu, \lambda)}\left|x_{\mu}-x_{\lambda}\right|\right)\left(1+2^{\min (\lambda, \nu)}\left|x_{\lambda}-x_{\nu}\right|\right)\right)^{N}}
\end{gathered}
$$

for some $C_{N}>0$.

Next we consider the situation where we are assuming cancellation and estimates for the derivatives. Fix $L$ to a nonnegative integer. (The condition for $|\gamma| \leq L-1$ below is vacuous when $L=0$.)

Proposition 4. Suppose that $\psi_{\nu}$ satisfies (20) and also

$$
\int_{\mathbf{R}^{n}} \psi_{\nu}(x) x^{\gamma} d x=0 \quad \text { for all }|\gamma| \leq L-1 .
$$

Let $\psi_{\mu, \lambda}$ be another function satisfying

$$
\left|\partial^{\gamma} \psi_{\mu, \lambda}(x)\right| \leq C_{N} \frac{2^{\mu n / 2} 2^{\lambda n / 2} \max \left(2^{\mu}, 2^{\lambda}\right)^{|\gamma|}}{\left(1+2^{\mu}\left|x-x_{\mu}\right|\right)^{N}\left(1+2^{\lambda}\left|x-x_{\lambda}\right|\right)^{N}} \quad \text { for all }|\gamma| \leq L
$$

for some $x_{\mu}, x_{\lambda}$ in $\mathbf{R}^{n}$, and all $N>n$. Suppose that $\nu \geq \max (\mu, \lambda)$. Then for all $N>n$ there exists a $C_{N, L}>0$ such that

$$
\begin{gathered}
\left|\int_{\mathbf{R}^{n}} \psi_{\nu}(x) \psi_{\mu, \lambda}(x) d x\right| \leq \\
\frac{C_{N, L} 2^{-(\nu-\max (\mu, \lambda)) L} 2^{-\nu n / 2} 2^{\mu n / 2} 2^{\lambda n / 2}}{\left(\left(1+2^{\min (\nu, \mu)}\left|x_{\nu}-x_{\mu}\right|\right)\left(1+2^{\min (\mu, \lambda)}\left|x_{\mu}-x_{\lambda}\right|\right)\left(1+2^{\min (\lambda, \nu)}\left|x_{\lambda}-x_{\nu}\right|\right)\right)^{N}} .
\end{gathered}
$$

Suppose now that the roles of $\psi_{\nu}$ and $\psi_{\mu, \lambda}$ are reversed. We have the following.

Proposition 5. Suppose that $\psi_{\nu}$ satisfies

$$
\left|\partial^{\gamma} \psi_{\nu}(x)\right| \leq C_{N, L} \frac{2^{\nu n / 2} 2^{\nu|\gamma|}}{\left(1+2^{\mu}\left|x-x_{\nu}\right|\right)^{N}} \quad \text { for all }|\gamma| \leq L
$$


for some $x_{\nu}$ in $\mathbf{R}^{n}$ and all $N>n$. Suppose also that $\psi_{\mu, \lambda}$ is another function satisfying (24) for $\gamma=0$ and

$$
\int_{\mathbf{R}^{n}} \psi_{\mu, \lambda}(x) x^{\gamma} d x=0 \quad \text { for all }|\gamma| \leq L-1 .
$$

Assume that $\max (\mu, \lambda) \geq \nu$. Then for all $N>0$ there exists a $C_{N, L}>0$ such that

$$
\begin{gathered}
\left|\int_{\mathbf{R}^{n}} \psi_{\nu}(x) \psi_{\mu, \lambda}(x) d x\right| \leq \\
\frac{C_{N, L} 2^{-(\max (\mu, \lambda)-\nu) L} 2^{-\max (\mu, \lambda) n / 2} 2^{\min (\mu, \lambda) n / 2} 2^{\nu n / 2}}{\left(\left(1+2^{\min (\nu, \mu)}\left|x_{\nu}-x_{\mu}\right|\right)\left(1+2^{\min (\mu, \lambda)}\left|x_{\mu}-x_{\lambda}\right|\right)\left(1+2^{\min (\lambda, \nu)}\left|x_{\lambda}-x_{\nu}\right|\right)\right)^{N}} .
\end{gathered}
$$

The proofs of Propositions 3, 4, and 5 involve some simple but long computations and they are postponed until the next section. The propositions above lead to a natural notion of bilinear molecules.

Definition 1. A collection of functions $\left\{\psi_{\mu l, \lambda m}\right\}$, with $\mu, \lambda \in \mathbf{Z}$ and $l, m \in \mathbf{Z}^{n}$ is called a family of bilinear smooth molecules if for all integers $N>0$ and all $\gamma$ multi-indices, there exist constants $C_{N, \gamma}$ such that

$$
\left|\partial^{\gamma} \psi_{\mu l, \lambda m}(x)\right| \leq C_{N, \gamma} \frac{2^{\mu n / 2} 2^{\lambda n / 2} \max \left(2^{\mu}, 2^{\lambda}\right)^{|\gamma|}}{\left(1+2^{\mu}\left|x-2^{-\mu} l\right|\right)^{N}\left(1+2^{\lambda}\left|x-2^{-\lambda} m\right|\right)^{N}}
$$

for all $N>n$ and

$$
\int_{\mathbf{R}^{n}} \psi_{\mu l, \lambda m}(x) d x=0
$$

for all $\mu, \lambda, l$, and $m$.

A consequence of Proposition 5 is that if a bilinear operator and its transposes map products of wavelets into bilinear molecules, then its associated tensor satisfies the almost diagonal condition of Theorem 1. Theorem 2 is then an easy consequence of Theorem 1 .

\section{Proofs of Propositions 3, 4, 5}

We begin with the following auxiliary lemma.

Lemma 1. Let $N>n$. There exists a constant $C$ depending only on the dimension $n$ and on $N$ such that for all $x_{0} \in \mathbf{R}^{n}$ and all $R>0$ we have

$$
b\left(x_{0}, R\right)=\int_{\left|x-x_{0}\right| \leq R}(1+|x|)^{-N} d x \leq C \frac{\max \left(R^{n}, R^{N}\right)}{\left(1+\left|x_{0}\right|\right)^{N}} .
$$

Proof. Let us suppose first that $R \leq 10$. Then consider the subcases $\left|x_{0}\right| \leq 20$ and $\left|x_{0}\right| \geq 20$. When $\left|x_{0}\right| \leq 20$ we obtain $\left|b\left(x_{0}, R\right)\right| \leq C R^{n}$ which implies (29) since $x_{0}$ is small. When $\left|x_{0}\right| \geq 20$ we have $|x| \approx\left|x_{0}\right|$ when $\left|x-x_{0}\right| \leq R \leq 10$ and hence the bound $b\left(x_{0}, R\right) \leq C R^{n}\left(1+\left|x_{0}\right|\right)^{-N}$ is easily obtained.

We now consider the case $R \geq 10$. In this case we split the region of integration in (29) into two subregions:

$$
\begin{aligned}
& A_{1}=\left\{x:|x| \geq\left|x_{0}\right| \text { and }\left|x-x_{0}\right| \leq R\right\}, \\
& A_{2}=\left\{x:|x| \leq\left|x_{0}\right| \text { and }\left|x-x_{0}\right| \leq R\right\} .
\end{aligned}
$$


The part of the integral in (29) over the set $A_{1}$ is easily shown to be bounded by $C R^{n}\left(1+\left|x_{0}\right|\right)^{-N}$ since $|x| \geq\left|x_{0}\right|$. It suffices to prove the required bound for the part of the integral in (29) over the set $A_{2}$. If $\left|x_{0}\right| \leq 2 R$ then

$$
\int_{A_{2}}(1+|x|)^{-N} d x \leq C^{\prime} \leq C \frac{R^{N}}{\left(1+\left|x_{0}\right|\right)^{N}} .
$$

If $\left|x_{0}\right| \geq 2 R$ we have $|x| \geq\left|x_{0}\right|-\left|x-x_{0}\right| \geq\left|x_{0}\right| / 2$ which allows us to estimate the part of the integral over $A_{2}$ in (29) by $C R^{n}\left(1+\frac{1}{2}\left|x_{0}\right|\right)^{N}$. This finishes the proof of Lemma 1 .

We continue with the proof of Proposition 3.

Proof. By symmetry we may assume that $\nu \geq \lambda \geq \mu$. Without loss of generality we may also assume that $x_{\mu}=0$. Next we observe that if the proposition is proved when $\mu=0$, then applying it to the functions $\psi_{\nu-\mu}, \psi_{\lambda-\mu}$, and $\psi_{0}$ centered at $x_{\nu-\mu}=2^{\mu} x_{\nu}, x_{\lambda-\mu}=2^{\mu} x_{\lambda}$, and $x_{0}=0$ gives the general case after a suitable change of variables.

We may therefore assume that $\nu \geq \lambda \geq \mu=0$ and that $x_{\mu}=0$. We start by writing the integral

$$
\int_{\mathbf{R}^{n}} \frac{2^{\nu n / 2}}{\left(1+2^{\nu}\left|x-x_{\nu}\right|\right)^{M}} \frac{2^{\lambda n / 2}}{\left(1+2^{\lambda}\left|x-x_{\lambda}\right|\right)^{M}} \frac{1}{(1+|x|)^{M}} d x
$$

as

$$
2^{(\lambda n+\nu n) / 2} \sum_{t=0}^{\infty} \sum_{s=0}^{\infty} 2^{-t M-s M} \int_{\substack{\left|x-x_{\nu}\right| \sim 2^{-\nu} 2^{t} \\\left|x-x_{\lambda}\right| \sim 2^{-\lambda} 2^{s}}}(1+|x|)^{-M} d x
$$

where the notation $\left|x-x_{\nu}\right| \sim 2^{-\nu} 2^{t}$ means $2^{-\nu} 2^{t} \leq\left|x-x_{\nu}\right| \leq 2^{-\nu} 2^{t+1}$ for $t \geq 1$ and $\left|x-x_{\nu}\right| \leq 2^{-\nu}$ when $t=0$. Next observe the following. If $\left|x-x_{\nu}\right| \sim 2^{-\nu} 2^{t}$ and $\left|x-x_{\lambda}\right| \sim 2^{-\lambda} 2^{s}$ then

$$
1+2^{\lambda}\left|x_{\nu}-x_{\lambda}\right| \leq 1+2^{\lambda+2} \max \left(2^{t-\nu}, 2^{s-\lambda}\right) \leq 8 \max \left(2^{t}, 2^{s}\right)
$$

and also

$$
\begin{aligned}
1+\left|x_{\lambda}\right| & \leq 1+\left|x_{\nu}-x_{\lambda}\right|+\left|x_{\nu}\right| \leq 1+4 \max \left(2^{t-\nu}, 2^{s-\lambda}\right)+\left|x_{\nu}\right| \\
& \leq 8 \max \left(2^{t}, 2^{s}\right)\left(1+\left|x_{\nu}\right|\right)
\end{aligned}
$$

Now use Lemma 1 to estimate

$$
\begin{aligned}
\int_{\substack{\left|x-x_{\nu}\right| \sim 2^{-\nu} 2^{t} \\
\left|x-x_{\lambda}\right| \sim 2^{-\lambda} 2^{s}}}(1+|x|)^{-M} d x & \leq C \frac{\max \left(2^{(t-\nu) n}, 2^{(t-\nu) 2 N}\right)}{\left(1+\left|x_{\nu}\right|\right)^{2 N}} \\
& \leq C \frac{\max \left(2^{(t-\nu) n}, 2^{(t-\nu) 2 N}\right)}{\left(1+\left|x_{\nu}\right|\right)^{N}\left(1+\left|x_{\lambda}\right|\right)^{N}} \max \left(2^{t}, 2^{s}\right)^{N}
\end{aligned}
$$


where we set $M=2 N$ and the second inequality above is a consequence of (B2). We now use this estimate to bound (30) by

$$
\begin{aligned}
& C 2^{(\lambda n+\nu n) / 2} \sum_{t=0}^{\infty} \sum_{s=0}^{\infty} 2^{-t M-s M} \frac{\max \left(2^{(t-\nu) n}, 2^{(t-\nu) 2 N}\right)}{\left(1+\left|x_{\nu}\right|\right)^{N}\left(1+\left|x_{\lambda}\right|\right)^{N}} \max \left(2^{t}, 2^{s}\right)^{N} \\
\leq & C 2^{(\lambda n+\nu n) / 2} \sum_{t=0}^{\infty} \sum_{s=0}^{\infty} 2^{-t M-s M} \frac{\max \left(2^{(t-\nu) n}, 2^{(t-\nu) 2 N}\right)}{\left(1+\left|x_{\nu}\right|\right)^{N}\left(1+\left|x_{\lambda}\right|\right)^{N}} \frac{\max \left(2^{t}, 2^{s}\right)^{2 N}}{\left(1+2^{\lambda}\left|x_{\nu}-x_{\lambda}\right|\right)^{N}} \\
\leq & C 2^{(\lambda n-\nu n) / 2}\left(1+\left|x_{\nu}\right|\right)^{-N}\left(1+\left|x_{\lambda}\right|\right)^{-N}\left(1+2^{\lambda}\left|x_{\nu}-x_{\lambda}\right|\right)^{-N}
\end{aligned}
$$

which proves the required estimate. We used (31) in the second estimate above and we took $M=5 N$ for the last series to converge.

We now continue with the proof of Proposition 4 .

Proof. We have

$$
\begin{aligned}
& \left|\int_{\mathbf{R}^{n}} \psi_{\nu}(x) \psi_{\mu, \lambda}(x) d x\right| \\
= & \left|\int_{\mathbf{R}^{n}} \psi_{\nu}(x)\left[\psi_{\mu, \lambda}(x)-\sum_{|\alpha| \leq L-1} \frac{\left(\partial^{\alpha} \psi_{\mu, \lambda}\right)\left(x_{\nu}\right)}{\alpha !}\left(x-x_{\nu}\right)^{\alpha}\right] d x\right| \\
\leq & \int_{\mathbf{R}^{n}}\left|\psi_{\nu}(x)\right|\left|\sum_{|\alpha|=L} \frac{\left(\partial^{\alpha} \psi_{\mu, \lambda}\right)(z)}{\alpha !}\left(x-x_{\nu}\right)^{\alpha}\right| d x \\
\leq & C \int_{\mathbf{R}^{n}}\left|\psi_{\nu}(x)\right| \frac{2^{\mu n / 2+\lambda n / 2+\max (\lambda, \mu) L}}{\left(1+2^{\mu}\left|z-x_{\mu}\right|\right)^{N}\left(1+2^{\lambda}\left|z-x_{\lambda}\right|\right)^{N}\left|x-x_{\nu}\right|^{L} d x} \\
\leq & C \int_{\mathbf{R}^{n}} \frac{2^{\mu n / 2+\lambda n / 2+\nu n / 2+\max (\lambda, \mu) L-\nu L}\left(\left|x-x_{\nu}\right| 2^{\nu}\right)^{L}}{\left(1+2^{\nu}\left|x-x_{\nu}\right|\right)^{N^{\prime}}\left(1+2^{\mu}\left|z-x_{\mu}\right|\right)^{N}\left(1+2^{\lambda}\left|z-x_{\lambda}\right|\right)^{N}} d x \\
\leq & C \int_{\mathbf{R}^{n}} \frac{2^{-(\nu-\max (\lambda, \mu)) L} 2^{\mu n / 2+\lambda n / 2+\nu n / 2}}{\left(1+2^{\nu}\left|x-x_{\nu}\right|\right)^{N^{\prime}-L}\left(1+2^{\mu}\left|z-x_{\mu}\right|\right)^{N}\left(1+2^{\lambda}\left|z-x_{\lambda}\right|\right)^{N}} d x \\
= & E_{\nu, \mu, \lambda}\left(x_{\nu}, x_{\mu}, x_{\lambda}\right),
\end{aligned}
$$

for some $z$ between $x$ and $x_{\nu}$. Now write the integral defining $E_{\nu, \mu, \lambda}\left(x_{\nu}, x_{\mu}, x_{\lambda}\right)$ as the sum of the integrals over the regions $A_{0}=\left\{x:\left|x-x_{\nu}\right| \leq 2^{-\nu}\right\}$ and $A_{j}=\left\{x: 2^{-\nu} 2^{j-1} \leq\left|x-x_{\nu}\right| \leq 2^{-\nu} 2^{j}\right\}$ for $j=1,2, \ldots$.

For $x \in A_{j}, j \geq 0$, we have that $\left|x-x_{\nu}\right| \leq 2^{j} 2^{-\nu}$. Then $|z-x| \leq\left|x_{\nu}-x\right|$ and hence

$$
\left|x-x_{\mu}\right| \leq|x-z|+\left|z-x_{\mu}\right| \leq\left|x-x_{\nu}\right|+\left|z-x_{\mu}\right| \leq 2^{j} 2^{-\nu}+\left|z-x_{\mu}\right| .
$$

Therefore

$$
\begin{aligned}
1+2^{\mu}\left|x-x_{\mu}\right| & \leq 1+2^{j} 2^{\mu-\nu}+2^{\mu}\left|z-x_{\mu}\right| \\
& \leq 1+2^{j}+2^{\mu}\left|z-x_{\mu}\right| \leq 2^{j+1}\left(1+2^{\mu}\left|z-x_{\mu}\right|\right) .
\end{aligned}
$$

Likewise we obtain

$$
1+2^{\lambda}\left|x-x_{\lambda}\right| \leq 2^{j+1}\left(1+2^{\lambda}\left|z-x_{\lambda}\right|\right) .
$$

These two estimates imply that when $x \in A_{0}$ we have

$$
\left(1+2^{\mu}\left|z-x_{\mu}\right|\right)^{-N} \leq C\left(1+2^{\mu}\left|x-x_{\mu}\right|\right)^{-N}
$$


and similarly

$$
\left(1+2^{\lambda}\left|z-x_{\lambda}\right|\right)^{-N} \leq C\left(1+2^{\lambda}\left|x-x_{\lambda}\right|\right)^{-N},
$$

while for $x \in A_{j}, j=1,2, \ldots$ we have

$$
\begin{aligned}
\left(1+2^{\mu}\left|z-x_{\mu}\right|\right)^{-N} & \leq C 2^{j N}\left(1+2^{\mu}\left|x-x_{\mu}\right|\right)^{-N} \\
& \leq C\left(2^{\nu}\left|x-x_{\nu}\right|\right)^{N}\left(1+2^{\mu}\left|x-x_{\mu}\right|\right)^{-N}
\end{aligned}
$$

and likewise

$$
\begin{aligned}
\left(1+2^{\lambda}\left|z-x_{\lambda}\right|\right)^{-N} & \leq C 2^{j N}\left(1+2^{\lambda}\left|x-x_{\lambda}\right|\right)^{-N} \\
& \leq C\left(2^{\nu}\left|x-x_{\nu}\right|\right)^{N}\left(1+2^{\lambda}\left|x-x_{\lambda}\right|\right)^{-N} .
\end{aligned}
$$

We now conclude that

$$
\begin{aligned}
& E_{\nu, \mu, \lambda}\left(x_{\nu}, x_{\mu}, x_{\lambda}\right) \\
= & \sum_{j=0}^{\infty} \int_{A_{j}} \frac{2^{\mu n / 2+\lambda n / 2+\nu n / 2} 2^{\max (\lambda, \mu) L-\nu L}}{\left(1+2^{\nu}\left|x-x_{\nu}\right|\right)^{N^{\prime}-L}\left(1+2^{\mu}\left|z-x_{\mu}\right|\right)^{N}\left(1+2^{\lambda}\left|z-x_{\lambda}\right|\right)^{N}} d x \\
\leq & C \sum_{j=0}^{\infty} \int_{A_{j}} \frac{2^{\mu n / 2+\lambda n / 2+\nu n / 2} 2^{\max (\lambda, \mu) L-\nu L}\left(2^{\nu}\left|x-x_{\nu}\right|\right)^{2 N}}{\left(1+2^{\nu}\left|x-x_{\nu}\right|\right)^{N^{\prime}-L}\left(1+2^{\mu}\left|x-x_{\mu}\right|\right)^{N}\left(1+2^{\lambda}\left|x-x_{\lambda}\right|\right)^{N}} d x \\
\leq & C \int_{\mathbf{R}^{n}} \frac{2^{\nu n / 2} 2^{\mu n / 2} 2^{\lambda n / 2} 2^{\max (\lambda, \mu) L-\nu L}}{\left(1+2^{\nu}\left|x-x_{\nu}\right|\right)^{N^{\prime}-L-2 N}\left(1+2^{\mu}\left|x-x_{\mu}\right|\right)^{N}\left(1+2^{\lambda}\left|x-x_{\lambda}\right|\right)^{N}} d x \\
\leq & C 2^{-(\nu-\max (\lambda, \mu)) L} \int_{\mathbf{R}^{n}} \frac{2^{\nu n / 2} 2^{\mu n / 2} 2^{\lambda n / 2}}{\left(1+2^{\nu}\left|x-x_{\nu}\right|\right)^{N}\left(1+2^{\mu}\left|x-x_{\mu}\right|\right)^{N}\left(1+2^{\lambda}\left|x-x_{\lambda}\right|\right)^{N}} d x
\end{aligned}
$$

where we picked $N^{\prime}>3 N+L$. Things now reduce to the situation discussed in the previous proposition except for the appearance the fixed factor $2^{-(\nu-\max (\lambda, \mu)) L}$.

Finally we discuss the proof of Proposition 5

Proof. For the proof below take $\mu \geq \lambda$ because of symmetry. We now use the cancellation on $\psi_{\nu}$ to subtract a suitable Taylor polynomial. We have

$$
\begin{aligned}
& \left|\int_{\mathbf{R}^{n}} \psi_{\mu, \lambda}(x) \psi_{\nu}(x) d x\right| \\
= & \left|\int_{\mathbf{R}^{n}} \psi_{\mu, \lambda}(x)\left[\psi_{\nu}(x)-\sum_{|\alpha| \leq L-1} \frac{\left(\partial^{\alpha} \psi_{\nu}\right)\left(x_{\mu}\right)}{\alpha !}\left(x-x_{\mu}\right)^{\alpha}\right] d x\right| \\
\leq & \int_{\mathbf{R}^{n}}\left|\psi_{\mu, \lambda}(x)\right|\left|\sum_{|\alpha|=L} \frac{\left(\partial^{\alpha} \psi_{\nu}\right)(z)}{\alpha !}\left(x-x_{\mu}\right)^{\alpha}\right| d x \\
\leq & C \int_{\mathbf{R}^{n}}\left|\psi_{\mu, \lambda}(x)\right| \frac{2^{\nu n / 2} 2^{\nu L}\left|x-x_{\mu}\right|^{L}}{\left(1+2^{\nu}\left|z-x_{\nu}\right|\right)^{N}} d x \\
\leq & C \int_{\mathbf{R}^{n}} \frac{2^{\mu n / 2}}{\left(1+2^{\mu}\left|x-x_{\mu}\right|\right)^{N_{1}}} \frac{2^{\lambda n / 2}}{\left(1+2^{\lambda}\left|x-x_{\lambda}\right|\right)^{N_{2}}} \frac{2^{\lambda n / 2} 2^{\nu L} 2^{-\mu L}\left(2^{\mu}\left|x-x_{\mu}\right|\right)^{L}}{\left(1+2^{\nu}\left|z-x_{\nu}\right|\right)^{N}} d x \\
\leq & C 2^{-(\mu-\nu) L} \int_{\mathbf{R}^{n}} \frac{2^{\lambda n / 2}}{\left(1+2^{\mu}\left|x-x_{\mu}\right|\right)^{N_{1}-L}} \frac{2^{\nu n / 2}}{\left(1+2^{\lambda}\left|x-x_{\lambda}\right|\right)^{N_{2}}} \frac{\left.2^{\mu n}\left|z-x_{\nu}\right|\right)^{N}}{\left(1+2^{\nu}\right.} d x \\
= & D_{\mu, \nu, \lambda}\left(x_{\mu}, x_{\nu}, x_{\lambda}\right),
\end{aligned}
$$


for some $z$ between $x$ and $x_{\mu}$. Now split $\mathbf{R}^{n}$ as the union of the sets $A_{j}$ where $A_{0}=\left\{x:\left|x-x_{\mu}\right| \leq 2^{-\mu}\right\}$ and $A_{j}=\left\{x: 2^{-\mu} 2^{j-1} \leq\left|x-x_{\mu}\right| \leq 2^{-\mu_{2}} 2^{j}\right.$ for $j=1,2, \ldots$

For $x \in A_{j}, j \geq 0$, we have $\left|x-x_{\mu}\right| \leq 2^{j} 2^{-\mu}$. Then $|z-x| \leq\left|x_{\mu}-x\right|$ and thus

$$
\left|x-x_{\nu}\right| \leq|x-z|+\left|z-x_{\nu}\right| \leq\left|x-x_{\mu}\right|+\left|z-x_{\nu}\right| \leq 2^{j} 2^{-\mu}+\left|z-x_{\mu}\right| .
$$

Therefore

$$
\begin{aligned}
1+2^{\nu}\left|x-x_{\nu}\right| & \leq 1+2^{j} 2^{\nu-\mu}+2^{\nu}\left|z-x_{\nu}\right| \\
& \leq 1+2^{j}+2^{\nu}\left|z-x_{\nu}\right| \leq 2^{j+1}\left(1+2^{\nu}\left|z-x_{\nu}\right|\right) .
\end{aligned}
$$

Now for $x \in A_{j}, j=1,2, \ldots$ we have

$$
\begin{aligned}
\left(1+2^{\nu}\left|z-x_{\nu}\right|\right)^{-N} & \leq C 2^{j N}\left(1+2^{\nu}\left|x-x_{\nu}\right|\right)^{-N} \\
& \leq C\left(2^{\mu}\left|x-x_{\mu}\right|\right)^{N}\left(1+2^{\nu}\left|x-x_{\nu}\right|\right)^{-N} .
\end{aligned}
$$

Now write

$$
\begin{aligned}
& D_{\mu, \nu, \lambda}\left(x_{\mu}, x_{\nu}, x_{\lambda}\right) \\
= & C 2^{-(\mu-\nu) L} \sum_{j=0}^{\infty} \int_{A_{j}} \frac{2^{\mu n / 2}}{\left(1+2^{\mu}\left|x-x_{\mu}\right|\right)^{N_{1}-L}} \frac{2^{\lambda n / 2}}{\left(1+2^{\lambda}\left|x-x_{\lambda}\right|\right)^{N_{2}}} \frac{2^{\nu n / 2}}{\left(1+2^{\nu}\left|z-x_{\nu}\right|\right)^{N}} d x \\
\leq & C 2^{-(\mu-\nu) L} \sum_{j=0}^{\infty} \int_{A_{j}} \frac{2^{\mu n / 2}\left(2^{\mu}\left|x-x_{\mu}\right|\right)^{N}}{\left(1+2^{\mu}\left|x-x_{\mu}\right|\right)^{N_{1}-L}} \frac{2^{\lambda n / 2}}{\left(1+2^{\lambda}\left|x-x_{\lambda}\right|\right)^{N_{2}}} \frac{2^{\nu n / 2}}{\left(1+2^{\nu}\left|x-x_{\nu}\right|\right)^{N}} d x \\
\leq & C 2^{-(\mu-\nu) L} \sum_{j=0}^{\infty} \int_{A_{j}} \frac{2^{\mu n / 2}}{\left(1+2^{\mu}\left|x-x_{\mu}\right|\right)^{N_{1}-L-N}} \frac{2^{\lambda n / 2}}{\left(1+2^{\lambda}\left|x-x_{\lambda}\right|\right)^{N_{2}}} \frac{2^{\nu n / 2}}{\left(1+2^{\nu}\left|x-x_{\nu}\right|\right)^{N}} d x \\
\leq & C 2^{-(\mu-\nu) L} \sum_{j=0}^{\infty} \int_{A_{j}} \frac{2^{\mu n / 2}}{\left(1+2^{\mu}\left|x-x_{\mu}\right|\right)^{N}} \frac{2^{\lambda n / 2}}{\left(1+2^{\lambda}\left|x-x_{\lambda}\right|\right)^{N}} \frac{2^{\nu n / 2}}{\left(1+2^{\nu}\left|x-x_{\nu}\right|\right)^{N}} d x,
\end{aligned}
$$

where we picked $N_{1}>2 N+L$ and $N_{2}=N$. We can now use Proposition 3 to complete the proof.

\section{The Proof of Theorem 1}

We now give the proof of our main result, Theorem 1

Proof. Fix a family of almost orthogonal wavelets $\left\{\phi_{\nu k}\right\}$ as in Section 2. For $f$ and $g$ in $\mathcal{S}_{0}$ the action of $T$ can be written as

$$
T(f, g)=\sum_{\lambda, m} \sum_{\mu, l} \sum_{\nu, k}\left\langle T\left(\phi_{\nu k}, \phi_{\mu l}\right), \phi_{\lambda m}\right\rangle\left\langle f, \phi_{\nu k}\right\rangle\left\langle g, \phi_{\mu l}\right\rangle \phi_{\lambda m}
$$

Let $u_{\nu k}=\left\langle f, \phi_{\nu k}\right\rangle, v_{\mu l}=\left\langle g, \phi_{\mu l}\right\rangle$, and $a(\lambda m, \nu k, \mu l)=\left\langle T\left(\phi_{\nu k}, \phi_{\mu l}\right), \phi_{\lambda m}\right\rangle$. Let us denote by

$$
S_{\lambda m}=\sum_{\mu, l} \sum_{\nu, k} a(\lambda m, \nu k, \mu l) u_{\nu k} v_{\mu l}
$$

We use the estimate

$$
\left|S_{\lambda m}\right| \leq \sum_{j=1}^{6}\left|S_{\lambda m}^{j}\right|
$$


where $S_{\lambda m}^{1}$ is the part of $S_{\lambda m}$ where $\nu \leq \mu \leq \lambda, S_{\lambda m}^{2}$ is the part of $S_{\lambda m}$ where $\mu \leq \nu \leq \lambda, S_{\lambda m}^{3}$ is the part of $S_{\lambda m}$ where $\nu \leq \lambda \leq \mu, S_{\lambda m}^{4}$ is the part of $S_{\lambda m}$ where $\mu \leq \lambda \leq \nu, S_{\lambda m}^{5}$ is the part of $S_{\lambda m}$ where $\lambda \leq \nu \leq \mu$, and $S_{\lambda m}^{6}$ is the part of $S_{\lambda m}$ where $\lambda \leq \mu \leq \nu$. We shall see that is enough to analyze $S_{\lambda m}^{1}$ and $S_{\lambda m}^{2}$.

Bound $S_{\lambda m}^{1}$ by a constant multiple of

$$
\sum_{\mu \leq \lambda} \sum_{\nu \leq \mu} \sum_{i=0}^{\infty} \sum_{j=0}^{\infty} \sum_{k} \sum_{l}\left|u_{\nu k}\right|\left|v_{\mu l}\right| \frac{2^{\frac{(\mu+\nu-\lambda) n}{2}} 2^{-(\lambda-\nu) \varepsilon} 2^{-i N} 2^{-j N}}{\left(1+2^{\nu}\left|2^{-\nu} k-2^{-\mu} l\right|\right)^{N}}
$$

where $k$ ranges over the set $I_{\nu \lambda m i}$ of all multi-indices in $\mathbf{Z}^{n}$ such that

$$
2^{\nu}\left|2^{-\nu} k-2^{-\lambda} m\right| \approx 2^{i}
$$

and $l$ ranges over the set $I_{\mu \lambda m j}$ of all multi-indices in $\mathbf{Z}^{n}$ such that

$$
2^{\mu}\left|2^{-\mu} l-2^{-\lambda} m\right| \approx 2^{j} .
$$

For a fixed $\lambda$ and $m$, let $Q_{\lambda m}$ be the unique dyadic cube in $\mathbf{R}^{n}$ of side-length $2^{-\lambda}$ whose lower left corner is the point $2^{-\lambda} m$. For $x \in Q_{\lambda m}$ we have $\left|x-2^{-\lambda} m\right| \leq$ $C 2^{-\lambda} \leq C 2^{-\nu}$, since we are assuming that $\lambda \geq \mu \geq \nu$. Also, since $\left|2^{-\nu} k-2^{-\lambda} m\right| \leq$ $C 2^{i-\nu}$, it follows that

$$
\left|x-2^{-\nu} k\right| \leq C 2^{i-\nu}
$$

for all $x \in Q_{\lambda m}$ and $k \in I_{\nu \lambda m i}$. Similarly, using $\lambda \geq \mu$ we obtain that

$$
\left|x-2^{-\mu} l\right| \leq C 2^{j-\mu}
$$

for all $x \in Q_{\lambda m}$ and $l \in I_{\mu \lambda m j}$.

Recall that for $Q_{\nu k}$ a dyadic cube in $\mathbf{R}^{n}, \widetilde{\chi}_{Q_{\nu k}}=2^{\nu n / 2} \chi_{Q_{\nu k}}$ is its $L^{2}$-normalized characteristic function. Fix now a $\nu, \mu, i$, and $j$ in (34) above and write

$$
\sum_{k \in I_{\nu \lambda m i}}\left|u_{\nu k}\right|=2^{\nu n / 2} \sum_{k \in I_{\nu \lambda m i}} \int_{Q_{\nu k}}\left|u_{\nu k}\right| \widetilde{\chi}_{Q_{\nu k}}(y) d y
$$

and similarly

$$
\sum_{l \in I_{\mu \lambda m j}}\left|v_{\mu l}\right|=2^{\mu n / 2} \sum_{l \in I_{\mu \lambda m j}} \int_{Q_{\mu l}}\left|v_{\mu l}\right| \widetilde{\chi}_{Q_{\mu l}}(y) d y .
$$

Next we observe that the union of dyadic cubes $Q_{\nu k}$ over all $k \in I_{\nu \lambda m i}$ in (37) is contained in a ball of measure $C 2^{(i-\nu) n}$. Similarly the union of all cubes in (38) is contained in a ball of measure $C 2^{(j-\mu) n}$. Now write

$$
\begin{aligned}
& M_{1}^{\nu}=M\left(\sum_{k}\left|u_{\nu k}\right| \widetilde{\chi}_{Q_{\nu k}}\right), \\
& M_{2}^{\mu}=M\left(\sum_{l}\left|v_{\mu l}\right| \widetilde{\chi}_{Q_{\mu l}}\right),
\end{aligned}
$$

where $M$ is the Hardy-Littlewood maximal function. We can then estimate the expression in (37) by

$$
C 2^{\nu n / 2} 2^{(i-\nu) n} M_{1}^{\nu}(x),
$$

and the expression in (38) by

$$
C 2^{\mu n / 2} 2^{(j-\mu) n} M_{2}^{\mu}(x)
$$


for any $x \in Q_{\lambda m}$. We now return to (34) which we can estimate by

$$
C \sum_{\mu \leq \lambda} \sum_{\nu \leq \mu} \sum_{i=0}^{\infty} \sum_{j=0}^{\infty} 2^{-(\lambda-\nu) \varepsilon} 2^{\frac{(\mu+\nu-\lambda) n}{2}} 2^{-i N} 2^{-j N} 2^{\frac{\nu n}{2}} 2^{\frac{\mu n}{2}} 2^{(i-\nu) n} M_{1}^{\nu}(x) 2^{(j-\mu) n} M_{2}^{\mu}(x)
$$

for any $x \in Q_{\lambda m}$. We therefore obtain that

$$
\left|S_{\lambda m}^{1} \chi_{Q_{\lambda m}}\right| \leq C \sum_{\mu \leq \lambda} \sum_{\nu \leq \mu} 2^{-(\lambda-\nu) \varepsilon} 2^{-\frac{\lambda n}{2}} M_{1}^{\nu} M_{2}^{\mu}
$$

or equivalently

$$
\left|S_{\lambda m}^{1} \widetilde{\chi}_{Q_{\lambda m}}\right| \leq C \sum_{\mu \leq \lambda} \sum_{\nu \leq \mu} 2^{-(\lambda-\nu) \varepsilon} M_{1}^{\nu} M_{2}^{\mu}
$$

We will now use this estimate to prove that

$$
\begin{aligned}
& \left\|\left(\sum_{\lambda}\left(\sum_{m}\left|S_{\lambda m}^{1}\right| \tilde{\chi}_{Q_{\lambda m}}\right)^{2}\right)^{1 / 2}\right\|_{L^{r}} \leq \\
& \quad C\left\|\left(\sum_{\nu}\left(\sum_{k}\left|u_{\nu k}\right| \widetilde{\chi}_{Q_{\nu k}}\right)^{2}\right)^{1 / 2}\right\|_{L^{p}}\left\|\left(\sum_{\mu}\left(\sum_{l}\left|v_{\mu l}\right| \widetilde{\chi}_{Q_{\mu l}}\right)^{2}\right)^{1 / 2}\right\|_{L^{q}} .
\end{aligned}
$$

Use 39 and the inequality $a b \leq a^{2}+b^{2}$ to estimate the square of the function in the $L^{r}$ norm inside the left hand side of (40) by

$$
C \sum_{\lambda} \sum_{\mu \leq \lambda} \sum_{\nu \leq \mu} \sum_{\mu^{\prime} \leq \lambda} \sum_{\nu^{\prime} \leq \mu^{\prime}} 2^{-(\lambda-\nu) \varepsilon} 2^{-\left(\lambda-\nu^{\prime}\right) \varepsilon}\left[\left(M_{1}^{\nu}\right)^{2}+\left(M_{1}^{\nu^{\prime}}\right)^{2}\right]\left[\left(M_{2}^{\mu}\right)^{2}+\left(M_{2}^{\mu^{\prime}}\right)^{2}\right] .
$$

By symmetry it suffices to bound only the term

$$
C \sum_{\lambda} \sum_{\mu \leq \lambda} \sum_{\nu \leq \mu} \sum_{\mu^{\prime} \leq \lambda} \sum_{\nu^{\prime} \leq \mu^{\prime}} 2^{-(\lambda-\nu) \varepsilon} 2^{-\left(\lambda-\nu^{\prime}\right) \varepsilon}\left(M_{1}^{\nu}\right)^{2}\left(M_{2}^{\mu}\right)^{2}
$$

and a similar term where $M_{2}^{\mu}$ is replaced by $M_{2}^{\mu^{\prime}}$ in (42). First sum over $\nu^{\prime} \leq \mu^{\prime}$ and then over $\mu^{\prime} \leq \lambda$ to estimate (42) by

$$
C \sum_{\lambda} \sum_{\mu \leq \lambda} \sum_{\nu \leq \mu} 2^{-(\lambda-\nu) \varepsilon}\left(M_{1}^{\nu}\right)^{2}\left(M_{2}^{\mu}\right)^{2}=C \sum_{\mu} \sum_{\lambda \geq \mu} \sum_{\nu \leq \mu} 2^{-(\lambda-\nu) \varepsilon}\left(M_{1}^{\nu}\right)^{2}\left(M_{2}^{\mu}\right)^{2}
$$

Next apply Fubini's theorem to change the order of summation and sum over $\lambda$ from $\mu$ to $\infty$. We conclude that (43), and thus (42) are bounded above by

$$
C\left(\sum_{\nu}\left(M_{1}^{\nu}\right)^{2}\right)\left(\sum_{\mu}\left(M_{2}^{\mu}\right)^{2}\right)
$$

We now consider the expression obtained when $M_{2}^{\mu}$ is replaced by $M_{2}^{\mu^{\prime}}$ in (42), that is

$$
\begin{aligned}
& C \sum_{\lambda} \sum_{\mu \leq \lambda} \sum_{\nu \leq \mu} \sum_{\mu^{\prime} \leq \lambda} \sum_{\nu^{\prime} \leq \mu^{\prime}} 2^{-(\lambda-\nu) \varepsilon} 2^{-\left(\lambda-\nu^{\prime}\right) \varepsilon}\left(M_{1}^{\nu}\right)^{2}\left(M_{2}^{\mu^{\prime}}\right)^{2} \\
& \quad=C \sum_{\lambda} \sum_{\nu \leq \lambda} \sum_{\mu^{\prime} \leq \lambda} \sum_{\nu^{\prime} \leq \mu^{\prime}} \sum_{\nu \leq \mu \leq \lambda} 2^{-(\lambda-\nu) \varepsilon} 2^{-\left(\lambda-\nu^{\prime}\right) \varepsilon}\left(M_{1}^{\nu}\right)^{2}\left(M_{2}^{\mu^{\prime}}\right)^{2} .
\end{aligned}
$$


First we sum over $\mu$ and then over $\nu^{\prime}$ in (44) to obtain the bound

$$
\begin{gathered}
C \sum_{\lambda} \sum_{\nu \leq \lambda} 2^{-(\lambda-\nu) \varepsilon}(\lambda-\nu) \sum_{\mu^{\prime} \leq \lambda} 2^{-\left(\lambda-\mu^{\prime}\right) \varepsilon}\left(M_{1}^{\nu}\right)^{2}\left(M_{2}^{\mu^{\prime}}\right)^{2} \\
\leq C \sum_{\nu}\left(M_{1}^{\nu}\right)^{2} \sum_{\lambda \geq \nu} 2^{-(\lambda-\nu) \varepsilon}(\lambda-\nu) \sum_{\mu^{\prime}}\left(M_{2}^{\mu^{\prime}}\right)^{2} .
\end{gathered}
$$

Next estimate $2^{-(\lambda-\nu) \varepsilon}(\lambda-\nu) \leq C$ in 45 and sum over $\lambda \in \mathbf{Z}$. Summing first in $\lambda$ we easily obtain the bound

$$
C\left(\sum_{\nu}\left(M_{1}^{\nu}\right)^{2}\right)\left(\sum_{\mu^{\prime}}\left(M_{2}^{\mu^{\prime}}\right)^{2}\right)
$$

for (45) and hence for (44).

We conclude that the square function inside the $L^{r}$ norm in (40) is bounded above by

$$
C\left(\sum_{\nu} M\left(\sum_{k}\left|u_{\nu k}\right| \widetilde{\chi}_{Q_{\nu k}}\right)^{2}\right)^{1 / 2}\left(\sum_{\mu} M\left(\sum_{l}\left|v_{\mu l}\right| \widetilde{\chi}_{Q_{\mu l}}\right)^{2}\right)^{1 / 2}
$$

We now estimate the $L^{r}$ norm of (46) using Hölder's inequality with exponents $1 / p+1 / q=1 / r$. We are then in a position to use the Fefferman-Stein vectorvalued maximal inequality (Theorem $\mathrm{C}$ ) to deduce the bound in (40). Observe that a simple interchange of the indices $\mu$ and $\nu$ gives that the term $S_{\lambda m}^{2}$ also satisfies estimate (40).

To treat $S_{\lambda m}^{j}$ for $3 \leq j \leq 6$ we use duality. Recall that $\dot{f}_{p}^{0,2}$ is the space of sequences $\left\{u_{\nu k}\right\}$ which satisfy

$$
\left\|\left(\sum_{\nu}\left(\sum_{k}\left|u_{\nu k}\right| \widetilde{\chi}_{Q_{\nu k}}\right)^{2}\right)^{1 / 2}\right\|_{L^{p}}<+\infty
$$

and that the dual space of $\dot{f}_{p}^{0,2}$ is $\dot{f}_{p^{\prime}}^{0,2}$, where $p^{\prime}=p /(p-1)$ for $1<p<\infty$. We have seen that the discrete operators $S_{\lambda m}^{j}, j=1,2$, map $\dot{f}_{p}^{0,2} \times \dot{f}_{q}^{0,2} \rightarrow \dot{f}_{r}^{0,2}$. This means that if $T$ has tensor $a(\lambda m, \nu k, \mu l)$ which satisfies $a(\lambda m, \nu k, \mu l)=0$ unless $\lambda \geq \mu \geq \nu$ or $\lambda \geq \nu \geq \mu$, then it must be bounded from $L^{p} \times L^{q} \rightarrow L^{r}$.

Let us denote by $A$ a tensor acting on sequences whose entries are $a(\lambda m, \nu k, \mu l)$. If $A$ is a bounded operator from $\dot{f}_{p}^{0,2} \times \dot{f}_{q}^{0,2} \rightarrow \dot{f}_{r}^{0,2}$, then $A^{* 1}$ is a bounded operator from $\dot{f}_{r^{\prime}}^{0,2} \times \dot{f}_{q}^{0,2} \rightarrow \dot{f}_{p^{\prime}}^{0,2}$ by duality. But as we have discussed earlier, the transpose $A^{* 1}$ has entries $a^{* 1}(\lambda m, \nu k, \mu l)$ given by

$$
a^{* 1}(\lambda m, \nu k, \mu l)=a(\nu k, \lambda m, \mu l) .
$$

Now, having proved that the tensor associated to $T$ is bounded in the case where $a(\lambda m, \nu k, \mu l)=0$ unless $\lambda \geq \mu \geq \nu$ or $\lambda \geq \nu \geq \mu$, we use duality as above to deduce the case where $\lambda$ and $\nu$ are interchanged, that is when $a(\lambda m, \nu k, \mu l)=0$ unless $\nu \geq \mu \geq \lambda$ or $\nu \geq \lambda \geq \mu$. (Note that we can use this duality argument because the hypotheses of the theorem are " $p$-independent", i.e. they are valid for all $1<p, q, r<\infty$ with $1 / p+1 / q=1 / r$.) Similarly we obtain the two remaining cases using the second transpose $A^{* 2}$. 
Finally, the boundedness of $T$ follows from the fact that

$$
\|T(f, g)\|_{L^{r}} \leq C \sum_{j=1}^{6}\left\|\left(\sum_{\lambda}\left(\sum_{m}\left|S_{\lambda m}^{j}\right| \widetilde{\chi}_{Q_{\lambda m}}\right)^{2}\right)^{1 / 2}\right\|_{L^{r}},
$$

and the wavelet characterization of $L^{p}$ spaces using the spaces $\dot{f}_{p}^{0,2}$.

\section{Almost diagonal CONDition for Other FUnCtion SPACES}

Our almost diagonal theorem can be extended to other function spaces. Here we will discuss the Triebel-Lizorkin spaces $\dot{F}_{p}^{\alpha, s}$ in the range $0<p<\infty, 1 \leq s \leq \infty$, and arbitrary $\alpha$. These spaces are defined by the quasi-norm

$$
\|f\|_{\dot{F}_{p}^{\alpha, s}}=\left\|\left(\sum_{\nu}\left(\left|f * \phi_{\nu}\right| 2^{\nu \alpha}\right)^{s}\right)^{1 / s}\right\|_{L^{p}\left(\mathbf{R}^{n}\right)},
$$

where $\phi$ is a smooth function as in Section 2 and $\phi_{\nu}(x)=2^{\nu n} \phi\left(2^{\nu} x\right)$. As usual, in the case $s=\infty$, the sup norm in $\nu$ is employed inside the $L^{p}$ norm in (47). These spaces should be interpreted as spaces of tempered distributions modulo polynomials but for particular values of the parameters they are identified with other classical spaces. The space $\mathcal{S}_{0}$ is dense in all $\dot{F}_{p}^{\alpha, s}$ and we have the duality $\left(\dot{F}_{p}^{\alpha, s}\right)^{\prime}=\dot{F}_{p^{\prime}}^{-\alpha, s^{\prime}}$, for $1<p, s<\infty$. In particular, $\dot{F}_{p}^{0,2} \approx L^{p}$ for $1<p<\infty$. For the same range of $p, \dot{F}_{p}^{\alpha, 2} \approx \dot{L}_{\alpha}^{p}$, the homogeneous Sobolev space of order $\alpha$. For $0<p \leq 1, \dot{F}_{p}^{0,2} \approx H^{p}$, the Hardy space in $\mathbf{R}^{n}$. Also, the "diagonal" spaces $\dot{F}_{p}^{\alpha, p}$ coincide with the Besov spaces $\dot{B}_{p}^{\alpha, p}$, often encountered, for example, in approximation theory. For details about Triebel-Lizorkin spaces see [22].

The Triebel-Lizorkin spaces admit wavelet decompositions as in Theorem A and the wavelet coefficients satisfy

$$
\|f\|_{\dot{F}_{p}^{\alpha, s}} \approx\left\|\left\{\left\langle f, \phi_{\nu k}\right\rangle\right\}\right\|_{\dot{f}_{p}^{\alpha, s}},
$$

where the spaces $\dot{f}_{p}^{\alpha, s}$ are the spaces of sequences $\left\{u_{\nu k}\right\}$ for which

$$
\left\|\left(\sum_{\nu} \sum_{k}\left(\left|u_{\nu k}\right| 2^{\nu \alpha} \widetilde{\chi}_{Q_{\nu k}}\right)^{s}\right)^{1 / s}\right\|_{L^{p}\left(\mathbf{R}^{n}\right)}<\infty .
$$

We are now ready to give some extensions of Theorem 1 to other function spaces. The $L^{p}$ case was treated separately for the sake of clarity in the presentation.

6.1. The case $s \neq 2$. In this case we have the following result.

Theorem 3. Suppose that the tensor $\{a(\lambda m, \nu k, \mu l)\}$ associated to a bilinear operator $T$ satisfies the same almost diagonal estimate of Theorem 1 . Then the corresponding operator $T$ can be extended to be bounded from $\dot{F}_{p}^{0, s_{1}} \times \dot{F}_{q}^{0, s_{2}}$ into $\dot{F}_{r}^{0, s_{3}}$ when $1<p, q, r<\infty, 1 / p+1 / q=1 / r, 1<s_{1}, s_{2} \leq \infty, 1 \leq s_{3}<\infty$ and $1 / s_{1}+1 / s_{2}=1 / s_{3}$.

Proof. As in the proof of Theorem 1 and with the same notation, we first need to establish

$$
\left(\sum_{\lambda}\left(\sum_{m}\left|S_{\lambda m}^{1}\right| \widetilde{\chi}_{Q_{\lambda m}}\right)^{s_{3}}\right)^{1 / s_{3}} \leq C\left(\sum_{\nu}\left(M_{1}^{\nu}\right)^{s_{1}}\right)^{1 / s_{1}}\left(\sum_{\mu}\left(M_{2}^{\mu}\right)^{s_{2}}\right)^{1 / s_{2}} .
$$


(With obvious modifications in the notation if one of the $s_{j}=\infty$.) We go back to (39) and obtain

$$
\begin{aligned}
& \left(\sum_{\lambda}\left(\sum_{m}\left|S_{\lambda m}^{1}\right| \tilde{\chi}_{Q_{\lambda m}}\right)^{s_{3}}\right)^{1 / s_{3}} \\
& \quad \leq C\left(\sum_{\lambda}\left(\sum_{\mu \leq \lambda} \sum_{\nu \leq \mu} 2^{-(\lambda-\nu) \varepsilon / 2} 2^{-(\mu-\nu) \varepsilon / 2} M_{1}^{\nu} M_{2}^{\mu}\right)^{s_{3}}\right)^{1 / s_{3}} .
\end{aligned}
$$

We write the inner double sum in the right hand side of (50) as

$$
\sum_{\mu} \sum_{\nu} K(\lambda, \nu, \mu) \chi_{\nu \leq \mu}(\nu, \mu) 2^{-(\mu-\nu) \varepsilon / 2} M_{1}^{\nu} M_{2}^{\mu},
$$

where

$$
K(\lambda, \nu, \mu)=2^{-(\lambda-\nu) \varepsilon / 2} \chi_{\mu \leq \lambda}(\lambda, \nu, \mu) \chi_{\nu \leq \mu}(\lambda, \nu, \mu) .
$$

The easy proof of the following is omitted.

\section{Lemma 2.}

$$
\sum_{\mu} \sum_{\nu} K(\lambda, \nu, \mu) \leq C \quad \text { for all } \lambda
$$

and

$$
\sum_{\lambda} K(\lambda, \nu, \mu) \leq C \quad \text { for all }(\nu, \mu)
$$

so the operator

$$
T(m)(\lambda)=\sum_{\mu} \sum_{\nu} K(\lambda, \nu, \mu) m(\nu, \mu)
$$

is bounded from $l^{s}(\nu, \mu)$ into $l^{s}(\lambda)$ for all $1 \leq s \leq \infty$.

Using Lemma 2 and (50) we obtain

$$
\left.\left(\sum_{\lambda} \sum_{m}\left|S_{\lambda m}^{1}\right| \widetilde{\chi}_{Q_{\lambda m}}\right)^{s_{3}}\right)^{1 / s_{3}} \leq C\left(\sum_{\mu} \sum_{\nu}\left(2^{-(\mu-\nu) \varepsilon / 2} \chi_{\nu \leq \mu}(\nu, \mu) M_{1}^{\nu} M_{2}^{\mu}\right)^{s_{3}}\right)^{1 / s_{3}} .
$$

We now apply Hölder's inequality with $1 / s_{1}+1 / s_{2}=1 / s_{3}$ to control the above by

$$
\left(\sum_{\nu} \sum_{\mu \geq \nu} 2^{-(\mu-\nu) \varepsilon_{1}}\left(M_{1}^{\nu}\right)^{s_{1}}\right)^{1 / s_{1}}\left(\sum_{\mu} \sum_{\nu \leq \mu} 2^{-(\mu-\nu) \varepsilon_{2}}\left(M_{2}^{\mu}\right)^{s_{2}}\right)^{1 / s_{2}}
$$

which leads to (49). We took $\varepsilon_{1}=\varepsilon s_{1} / 4$ and $\varepsilon_{2}=\varepsilon s_{2} / 4$ in (52) above.

As in Theorem 11, the bounds for $S_{\lambda m}^{2}$ follow by simply interchanging the roles of $\mu$ and $\nu$. To obtain the estimates for the remaining cases, we observe that if a tensor $A$ is bounded from $\dot{f}_{p}^{0, s_{1}} \times \dot{f}_{q}^{0, s_{2}}$ into $\dot{f}_{r}^{0, s_{3}}$ for all $1 / p+1 / q=1 / r$, $1 / s_{1}+1 / s_{2}=1$ then its first transpose $A^{* 1}$ is bounded from $\dot{f}_{r^{\prime}}^{0, s_{3}^{\prime}} \times \dot{f}_{q}^{0, s_{2}}$ into $\dot{f}_{p^{\prime}}^{0, s_{1}^{\prime}}$, where $1 / r^{\prime}+1 / q=1 / p^{\prime}$ and $1 / s_{3}^{\prime}+1 / s_{2}=1 / s_{1}^{\prime}$. Note also that the restrictions on the $s_{j}$ 's in the statement of the theorem translate into $1<s_{3}^{\prime}, s_{2} \leq \infty$ and $1 \leq s_{1}^{\prime}<\infty$. Similarly with $A^{* 2}$. Finally, the same duality arguments used in Theorem 1 now complete the proof.

Note that the restrictions $s_{1}, s_{2}>1$ in this proof are imposed by Theorem $\mathrm{C}$. 
Observe that by taking $s_{1}=s_{2}=2$, Theorem 3 actually gives a sharper version of Theorem 1. In fact, if $T$ satisfies the almost diagonal condition of Theorem 1$]$ then $T$ must map $L^{p} \times L^{q} \rightarrow \dot{F}_{r}^{0,1}$ which is a proper subspace of $L^{r}=\dot{F}_{r}^{0,2}$.

The following is a corollary of Theorem 3 ,

Corollary 1. Under the hypotheses of Theorem 3, T extends to a bounded operator from $\dot{F}_{p}^{0, s_{1}} \times \dot{F}_{q}^{0, s_{2}}$ into $\dot{F}_{r}^{0, \min \left(s_{1}, s_{2}\right)}$.

Proof. Note that $s_{3} \leq \min \left(s_{1}, s_{2}\right)$. The trivial embeddings $\dot{F}_{p}^{0, s} \subset \dot{F}_{p}^{0, t}$ and $\dot{f}_{p}^{0, s} \subset$ $\dot{f}_{p}^{0, t}$ when $s<t$ imply the required conclusion.

6.2. The case $p \leq 1$. The following result gives, in particular, boundedness of a bilinear operator on a product of Hardy spaces $H^{p}=\dot{F}_{p}^{0,2}$, for $0<p \leq 1$.

Theorem 4. Let $\{a(\lambda m, \nu k, \mu l)\}$ be the tensor associated to a bilinear operator $T$. Let $0<p, q, r<\infty, 1 / p+1 / q=1 / r, 1<s_{1}, s_{2} \leq \infty, 1 \leq s_{3}<\infty, 1 / s_{1}+1 / s_{2}=$ $1 / s_{3}$, and fix $t<\min (1, p, q, r)$. Suppose that

$$
|a(\lambda m, \nu k, \mu l)| \leq
$$

$C 2^{-(\max (\mu, \nu, \lambda)-\min (\mu, \nu, \lambda)) \varepsilon / t} 2^{(-\max (\mu, \nu, \lambda)+\operatorname{med}(\mu, \nu, \lambda)+\min (\mu, \nu, \lambda)) n / 2 t} 2^{(\lambda-\nu-\mu) n(t-1) / 2 t}$

for some $C>0, N>n$, and $\varepsilon>0$. Then the operator $T$ can be extended to be $a$ bounded operator from $\dot{F}_{p}^{0, s_{1}} \times \dot{F}_{q}^{0, s_{2}}$ into $\dot{F}_{r}^{0, s_{3}}$.

Proof. For sequence $u=\left\{u_{\nu k}\right\}$ let $\widetilde{u}=\left\{\widetilde{u}_{\nu k}\right\}=\left\{2^{\nu n(1-t) / 2}\left|u_{\nu k}\right|^{t}\right\}$. It is easy to see that

$$
\|\widetilde{u}\|_{\dot{f}_{p / t}^{0, s / t}}^{1 / t}=\|u\|_{\dot{f}_{p}^{0, s}}
$$

Given this observation, the result that we are trying to prove can be reduced to previous cases. Note that by the choice of $t$, we have $p / t, q / t, r / t>1$. Let $A$ be the tensor associated with $T$ and let $B$ be the tensor with entries given by

$$
b(\lambda m, \nu k, \mu l)=|a(\lambda m, \nu k, \mu l)|^{t} 2^{-\lambda n(t-1) / 2} 2^{\nu n(t-1) / 2} 2^{\mu n(t-1) / 2} .
$$

Since $t<1$, for any pair of sequences $u=\left\{u_{\nu k}\right\}$ and $v=\left\{v_{\mu l}\right\}$ we have

$$
\begin{gathered}
\left|A(u, v)_{\lambda m}\right| \leq\left(\sum_{\mu l} \sum_{\nu k}|a(\lambda m, \nu k, \mu l)|^{t}\left|u_{\nu k}\right|^{t}\left|v_{\mu l}\right|^{t}\right)^{1 / t} \\
=\left(2^{\lambda n(t-1) / 2} \sum_{\mu l} \sum_{\nu k} b(\lambda m, \nu k, \mu l) 2^{-\nu n(t-1) / 2} 2^{-\mu n(t-1) / 2}\left|u_{\nu k}\right|^{t}\left|v_{\mu l}\right|^{t}\right)^{1 / t} .
\end{gathered}
$$

Observe that the tensor $B$ satisfies the hypotheses of Theorem 3 . Using (53) and the boundedness of $B$ we get that

$$
\begin{gathered}
\|A(u, v)\|_{\dot{f}_{r}^{0, s_{3}}} \leq\|B(\widetilde{u}, \widetilde{v})\|_{\dot{f}_{r / t}^{0, s_{3} / t}}^{1 / t} \\
\leq C\|\widetilde{u}\|_{\dot{f}_{p / t}^{0, s_{1} / t}}^{1 / t}\|\widetilde{v}\|_{\dot{f}_{q}^{0, s_{2} / t} / t}^{1 / t} \\
\leq C\|u\|_{\dot{f}_{p}^{0, s_{1}}}\|v\|_{\dot{f}_{q}^{0, s_{2}} .}
\end{gathered}
$$


6.3. The case $\alpha \neq 0$. This case includes results for (homogeneous) Sobolev spaces $\dot{L}_{\alpha}^{p}=\dot{F}_{p}^{\alpha, 2}$.

Theorem 5. Let $\{a(\lambda m, \nu k, \mu l)\}$ be the tensor associated to a bilinear operator $T$. Let $0<p, q, r<\infty, 1 / p+1 / q=1 / r, 1<s_{1}, s_{2}, s_{3}<\infty, 1 / s_{1}+1 / s_{2}=1 / s_{3}$, let $\alpha_{3}=\alpha_{1}+\alpha_{2}$ for any $\alpha_{1}, \alpha_{2}, \alpha_{3}$ and fix $t<\min (1, p, q, r)$. Suppose that

$$
\begin{gathered}
2^{\left(\lambda \alpha_{3}-\nu \alpha_{2}-\mu \alpha_{1}\right) / t}|a(\lambda m, \nu k, \mu l)| \leq \\
\frac{C 2^{-(\max (\mu, \nu, \lambda)-\min (\mu, \nu, \lambda)) \varepsilon / t} 2^{(-\max (\mu, \nu, \lambda)+\operatorname{med}(\mu, \nu, \lambda)+\min (\mu, \nu, \lambda)) n / 2 t} 2^{(\lambda-\nu-\mu) n(t-1) / 2 t}}{\left(\left(1+2^{\min (\nu, \mu)}\left|2^{-\nu} k-2^{-\mu} l\right|\right)\left(1+2^{\min (\mu, \lambda)}\left|2^{-\mu} l-2^{-\lambda} m\right|\right)\left(1+2^{\min (\lambda, \nu)}\left|2^{-\lambda} m-2^{-\nu} k\right|\right)\right)^{N / t}}
\end{gathered}
$$

for some $C>0, N>n$, and $\varepsilon>0$. Then the operator $T$ can be extended to be a bounded operator from $\dot{F}_{p}^{\alpha_{1}, s_{1}} \times \dot{F}_{q}^{\alpha_{2}, s_{2}}$ into $\dot{F}_{r}^{\alpha_{3}, s_{3}}$.

Proof. The theorem reduces to the previous cases with $\alpha=0$ by observing now that for $\widetilde{u}=\left\{\widetilde{u}_{\nu k}\right\}=\left\{2^{\nu \alpha} u_{\nu k}\right\}$.

$$
\|\widetilde{u}\|_{\dot{f}_{p}^{0, s}}=\|u\|_{\dot{f}_{p}^{\alpha, s}}
$$

We leave the simple computations to the reader.

\section{EXAMPLES AND APPLICATIONS}

We now look at some examples given by bilinear operators defined by their symbols. First we consider general symbols $\sigma(x, \xi, \eta)$ which are $C^{\infty}$ functions on $\mathbf{R}^{n} \times\left(\mathbf{R}^{n}-\{0\}\right) \times\left(\mathbf{R}^{n}-\{0\}\right)$ and satisfy the homogeneous estimates

$$
\left|\partial_{x}^{\gamma} \partial_{\xi}^{\beta} \partial_{\eta}^{\delta} \sigma(x, \xi, \eta)\right| \leq C_{\gamma, \beta, \delta}|\xi|^{-|\beta|}|\eta|^{-|\delta|}(|\xi|+|\eta|)^{|\gamma|}
$$

for all $x, \xi \neq 0, \eta \neq 0$ and all multi-indices $\gamma, \beta$ and $\delta$. Example of such symbols are the often encountered "homogeneous multipliers", that is functions $\sigma(\xi, \eta)$ independent of $x$ and homogeneous of degree zero in $\xi$ and in $\eta$. We have the following estimate for the action of such bilinear operators on pairs of wavelets.

Lemma 3. Let $T$ be a bilinear operator with symbol $\sigma(x, \xi, \eta)$ satisfying (55). Then, for any family of almost orthogonal wavelets $\left\{\phi_{\nu k}\right\}$ as in Section 2,

$$
\left|\partial^{\gamma} T\left(\phi_{\nu k}, \phi_{\mu l}\right)(x)\right| \leq C_{N, \gamma} \frac{2^{\nu n / 2} 2^{\mu n / 2} \max \left(2^{\nu}, 2^{\mu}\right)^{|\gamma|}}{\left(1+2^{\nu}\left|x-2^{-\nu} k\right|\right)^{N}\left(1+2^{\mu}\left|x-2^{-\mu} l\right|\right)^{N}}
$$

for all $\gamma$ and all $N>n$.

Proof. With a simple change of variable, we compute that

$$
T\left(\phi_{\nu k}, \phi_{\mu l}\right)(x)=2^{\nu n / 2} 2^{\mu n / 2} \iint e^{i\left(\left(2^{\nu} x-k\right) \xi+\left(2^{\mu} x-l\right) \eta\right)} \sigma\left(x, 2^{\nu} \xi, 2^{\mu} \eta\right) \widehat{\phi}(\xi) \widehat{\phi}(\eta) d \xi d \eta
$$

where the integration takes place for $|\xi| \approx 1$ and $|\eta| \approx 1$ because of the conditions on the support of $\widehat{\phi}$. Taking $\gamma$ derivatives in $x$ we obtain a sum of terms with integrands of the form

$$
C_{\gamma_{1}, \gamma_{2}} e^{i\left(\left(2^{\nu} x-k\right) \xi+\left(2^{\mu} x-l\right) \eta\right)}\left(i\left(2^{\nu} \xi+2^{\mu} \eta\right)\right)^{\gamma_{1}} \partial_{x}^{\gamma_{2}} \sigma\left(x, 2^{\nu} \xi, 2^{\mu} \eta\right) \widehat{\phi}(\xi) \widehat{\phi}(\eta)
$$

where $\gamma_{1}+\gamma_{2}=\gamma$. 
Now, if $\triangle_{\xi}$ is the Laplace operator in $\xi$, then

$$
\left(1-\triangle_{\xi}\right)^{N} e^{i\left(\left(2^{\nu} x-k\right) \xi+\left(2^{\mu} x-l\right) \eta\right)}=\left(1+\left|2^{\nu} x-k\right|\right)^{N} e^{i\left(\left(2^{\nu} x-k\right) \xi+\left(2^{\mu} x-l\right) \eta\right)},
$$

and integration by parts in $\xi$ gives

$$
\partial^{\gamma} T\left(\phi_{\nu k}, \phi_{\mu l}\right)(x)=\frac{2^{\nu n / 2} 2^{\mu n / 2}}{\left(1+\left|2^{\nu} x-k\right|\right)^{N}} \iint e^{i\left(\left(2^{\nu} x-k\right) \xi+\left(2^{\mu} x-l\right) \eta\right)} \sum_{\gamma_{1}, \gamma_{2}, \beta_{1}, \beta_{2}, \beta_{3}} d \xi d \eta
$$

where $\sum_{\gamma_{1}, \gamma_{2}, \beta_{1}, \beta_{2}, \beta_{3}}$ is a finite sum with $\beta_{1}+\beta_{2}+\beta_{3}=\beta$ and $|\beta| \leq 2 N$, and each of the summands is of the form

$$
C_{\gamma_{1}, \gamma_{2}, \beta_{1}, \beta_{2}, \beta_{3}} \partial_{\xi}^{\beta_{1}}\left(2^{\nu} \xi+2^{\mu} \eta\right)^{\gamma_{1}} \partial_{\xi}^{\beta_{2}} \partial_{x}^{\gamma_{2}} \sigma\left(x, 2^{\nu} \xi, 2^{\mu} \eta\right) \partial_{\xi}^{\beta_{3}} \widehat{\phi}(\xi) \widehat{\phi}(\eta)
$$

Using the size of $\xi$ and $\eta$ and the conditions on the symbol we obtain the simple estimates

$$
\begin{gathered}
\left|\partial_{\xi}^{\beta_{1}}\left(2^{\nu} \xi+2^{\mu} \eta\right)^{\gamma_{1}}\right| \leq C_{\gamma_{1}, \beta_{1}} \max \left(2^{\nu}, 2^{\mu}\right)^{\left|\gamma_{1}\right|} \\
\left|\partial_{\xi}^{\beta_{2}} \partial_{x}^{\gamma_{2}} \sigma\left(x, 2^{\nu} \xi, 2^{\mu} \eta\right)\right| \\
\leq C_{\gamma_{2}, \beta_{2}}\left|2^{\nu} \xi\right|^{-\left|\beta_{2}\right|} 2^{\nu\left|\beta_{2}\right|}\left(2^{\nu}|\xi|+2^{\mu}|\eta|\right)^{\left|\gamma_{2}\right|} \\
\leq C_{\gamma_{2}, \beta_{2}} \max \left(2^{\nu}, 2^{\mu}\right)^{\left|\gamma_{2}\right|}
\end{gathered}
$$

and

$$
\left|\partial_{\xi}^{\beta_{3}} \widehat{\phi}(\xi) \widehat{\phi}(\eta)\right| \leq C_{\beta_{3}} .
$$

Combining (56), (57), and (58) we obtain

$$
\left|\partial^{\gamma} T\left(\phi_{\nu k}, \phi_{\mu l}\right)(x)\right| \leq C_{N, \gamma} \frac{2^{\nu n / 2} 2^{\mu n / 2}}{\left(1+\left|2^{\nu} x-k\right|\right)^{N}} \max \left(2^{\nu}, 2^{\mu}\right)^{\gamma} .
$$

The same computations using integration by parts in the variable $\eta$ give

$$
\left|\partial^{\gamma} T\left(\phi_{\nu k}, \phi_{\mu l}\right)(x)\right| \leq C_{N, \gamma} \frac{2^{\nu / 2} 2^{\mu / 2}}{\left(1+\left|2^{\mu} x-l\right|\right)^{N}} \max \left(2^{\nu}, 2^{\mu}\right)^{\gamma} .
$$

Estimates (59) and (60) together yield the desired result.

The above lemma implies a "reduced" $T 1$-theorem for bilinear pseudodifferential operators.

Theorem 6. Let $T$ be a bilinear operator. Assume that the symbols of $T, T^{* 1}, T^{* 2}$ satisfy (55). Assume further that for a family of wavelets $\left\{\phi_{\nu k}\right\}$

$$
\int T\left(\phi_{\nu k}, \phi_{\mu l}\right)(x) d x=\int T^{* 1}\left(\phi_{\nu k}, \phi_{\mu l}\right)(x) d x=\int T^{* 2}\left(\phi_{\nu k}, \phi_{\mu l}\right)(x) d x=0 .
$$

Then $T$ can be extended as a bounded operator from $L^{p}\left(\mathbf{R}^{n}\right) \times L^{q}\left(\mathbf{R}^{n}\right)$ into $L^{r}\left(\mathbf{R}^{n}\right)$ when $1 / p+1 / q=1 / r$ and $1<p, q, r<\infty$.

Proof. We just observe that as a consequence of Lemma 3, $T, T^{* 1}, T^{* 2}$, map pairs of wavelets into bilinear molecules. The result then follows from Theorem 2

Replacing (61) by higher order vanishing moments one can obtain versions of the theorem above for other function spaces. We illustrate this below.

The cancellation conditions in (61) take a very simple form for bilinear multipliers, i.e. when there is no $x$-dependence in the symbol. In fact, it is easy to see that

$$
\int T\left(\phi_{\nu k}, \phi_{\mu l}\right)(x) x^{\gamma} d x=0
$$


for all $|\gamma| \leq L$ is equivalent to

$$
\partial_{\xi}^{\gamma}(\sigma(\xi,-\xi))=0
$$

for all $|\gamma| \leq L$. Similarly, the corresponding higher order cancellations for $T^{* 1}$ and $T^{* 2}$ are equivalent to

$$
\partial_{\xi}^{\gamma}(\sigma(\xi, 0))=0
$$

and

$$
\partial_{\xi}^{\gamma}(\sigma(0, \xi))=0
$$

for all $|\gamma| \leq L$.

We can now extend the $L^{p}$ multiplier result of Coifman and Meyer [5] to $H^{p}$ spaces in the full range $0<p \leq 1$. See also the works of Coifman, Dobyinsky, and Meyer [2] and of Youssfi [23]. In the theorem below we identify $H^{p}$ with $L^{p}$ for $p>1$.

Theorem 7. Let $\sigma(\xi, \eta)$ be a $C^{\infty}$ function on $\mathbf{R}^{n} \times \mathbf{R}^{n}-\{(0,0)\}$ such that

$$
\left|\partial_{\xi}^{\gamma} \partial_{\eta}^{\beta} \sigma(\xi, \eta)\right| \leq C_{\gamma, \beta}(|\xi|+|\eta|)^{-|\gamma|-|\beta|}
$$

for all $\xi \neq 0, \eta \neq 0$ and all multi-indices $\gamma$ and $\beta$. Assume that $0<p, q, r<\infty$, $1 / p+1 / q=1 / r$, and $r \leq 1$. In addition, assume that $\sigma$ satisfies (63), 64), (65), with $L=\left[n\left(\frac{1}{r}-1\right)\right]+1$. Then the corresponding bilinear operator $T$ with symbol $\sigma$ is bounded from $H^{p} \times H^{q}$ into $\dot{F}_{r}^{0,1} \subset H^{r}$.

Proof. It is easy to see that the class of operators with symbols satisfying (66) is invariant by taking either transpose. Hence, Lemma 3 can be applied to $T, T^{* 1}$, and $T^{* 2}$. We will use this and Proposition 5 to verify that $T$ satisfies the estimate in the hypotheses of Theorem 4 To do this, fix $t<\min (1, p, q, r)=r$ and let

$$
\begin{aligned}
& B_{N}=\left(\left(1+2^{\min (\nu, \mu)}\left|2^{-\nu} k-2^{-\mu} l\right|\right)\right.\left(1+2^{\min (\mu, \lambda)}\left|2^{-\mu} l-2^{-\lambda} m\right|\right) \\
&\left.\times\left(1+2^{\min (\lambda, \nu)}\left|2^{-\lambda} m-2^{-\nu} k\right|\right)\right)^{N} .
\end{aligned}
$$

By symmetry, we may assume $\mu \geq \nu$ and consider the following three cases.

a) $\lambda \geq \mu \geq \nu$. Using Proposition 5 with $T^{* 1}\left(\phi_{\lambda m}, \phi_{\mu l}\right)$ and $\phi_{\nu k}$, we obtain

$$
\begin{aligned}
|a(\lambda m, \nu k, \mu l)| & \leq \frac{C}{B_{N}} 2^{-(\lambda-\nu) L} 2^{(-\lambda+\mu+\nu) n / 2} \\
& \leq \frac{C}{B_{N}} 2^{-(\lambda-\nu) \epsilon / t} 2^{(-\lambda+\mu+\nu) n(t-1) / 2 t} 2^{(-\lambda+\mu+\nu) n / 2 t},
\end{aligned}
$$

when $L \geq \epsilon / t$.

b) $\mu \geq \lambda \geq \nu$. Apply again Proposition 5 to now obtain

$$
\begin{aligned}
|a(\lambda m, \nu k, \mu l)| & \leq \frac{C}{B_{N}} 2^{-(\mu-\nu) L} 2^{(-\mu+\lambda+\nu) n / 2} \\
& \leq \frac{C}{B_{N}} 2^{-(\mu-\nu) \epsilon / t} 2^{(-\lambda+\mu+\nu) n(t-1) / 2 t} 2^{(-\mu+\lambda+\nu) n / 2 t},
\end{aligned}
$$

when $L \geq \epsilon / t+n(1 / t-1)$. 
c) $\mu \geq \nu \geq \lambda$. This time Proposition [5 with $T\left(\phi_{\nu k}, \phi_{\mu l}\right)$ and $\phi_{\lambda m}$ gives

$$
\begin{aligned}
|a(\lambda m, \nu k, \mu l)| & \leq \frac{C}{B_{N}} 2^{-(\mu-\lambda) L} 2^{(-\mu+\lambda+\nu) n / 2} \\
& \leq \frac{C}{B_{N}} 2^{-(\mu-\lambda) \epsilon / t} 2^{(-\lambda+\mu+\nu) n(t-1) / 2 t} 2^{(-\mu+\lambda+\nu) n / 2 t}
\end{aligned}
$$

again when $L \geq \epsilon / t+n(1 / t-1)$.

By selecting $\epsilon>0$ sufficiently small and $t<r$ sufficiently close to $r$, we obtain the requirement $L \geq n\left(\frac{1}{r}-1\right)+\delta$ for $\delta>0$ arbitrarily small. This justifies the choice of $L=\left[n\left(\frac{1}{r}-1\right)\right]+1$ in the statement of the theorem. To complete the proof we pick $N$ so that $N t>n$ and we apply Theorem 4 .

\section{Concluding Remarks}

Using these methods, it is possible to consider not only bilinear but also more general $k$-linear operators for $k \geq 3$. Nevertheless, having preferred to minimize the cumbersome notation and for the sake of clarity in the exposition, we have limited ourselves to the bilinear case. We also note that the proofs in this article do not require the symbols or molecules to be $C^{\infty}$, but only $C^{k}$ for sufficiently large $k$ depending on the parameters involved. We find no need in this work to state the amount of smoothness required in the proofs.

As in the introduction, we remark that the almost diagonal conditions are $p$ independent provided $1<p<\infty$. This is an indication that our results should apply to a larger class of bilinear operators defined by singular kernels. We have in mind kernels which satisfy bilinear analogues of the standard estimates of linear operators of Calderón-Zygmund type. We plan to address this issue in a forthcoming collaboration.

In general, the almost diagonal conditions are only sufficient to get boundedness results. Nevertheless, when combined with appropriate cancellation they become very close to also being necessary for certain classes of operators (again analogues of the linear Calderón-Zygmund operators). For example, Theorem 7 with $p=q=2$ gives the boundedness of $T$ from $L^{2} \times L^{2}$ into $\dot{F}_{1}^{0,1}$. This last space coincides with the Besov ("special atoms") space $\dot{B}_{1}^{0,1}$. It has been proved in 2 that in this particular case the hypotheses of Theorem 7 are necessary and sufficient for such a boundedness result. The other cases treated in Theorem 7 are new. We do not know whether the cancellation hypotheses are also necessary.

Examples of operators to which Theorem 7 applies are provided by homogeneous multipliers of degree zero in $\mathbf{R}^{2 n}$ which are smooth on the unit sphere and vanish in a neighborhood of $\xi=-\eta, \xi=0$, and $\eta=0$. A different approach to homogeneous bilinear multipliers has been pursued by Gilbert and Nahmod [12] using tiles in the spirit of the work [17]. The methods in [12] treat bilinear multipliers with less regularity than the ones we are considering but so far the techniques using tiles are only available in dimension one.

The interested reader may check that, with the appropriate amount of cancellation on $T$ and its transposes, the method of this article gives versions of Theorem 7 for other Triebel-Lizorkin spaces $\dot{F}_{p}^{\alpha, s}$. Also by combining with the $L^{p}$ results in [5] one can obtain versions for the inhomogeneous spaces $F_{p}^{\alpha s}=L^{p} \cap \dot{F}_{p}^{\alpha s}$ for $1<p<\infty$ and $\alpha>0$. Besov spaces can also be considered. However for those 
spaces there is a more direct alternative approach based on a multilinear version of Schur's test. Details will appear elsewhere [14].

\section{REFERENCES}

[1] G. Bourdaud, Une algèbre maximale d'opérateurs pseudodifferentiels, Comm. Partial Diff. Eq. 13 (1988), 1059-1083. MR 89g:47063

[2] R. R. Coifman, S. Dobyinsky, and Y. Meyer, Opérateurs bilinéaires et renormalization, in Essays on Fourier Analysis in Honor of Elias M. Stein, C. Fefferman, R. Fefferman, S. Wainger (eds), Princeton University Press, Princeton NJ, 1995. MR 96b:42019]

[3] R. R. Coifman and L. Grafakos, Hardy space estimates for multilinear operators, Rev. Mat. Iberoamer. 8 (1992), 45-67. MR 93j:42011

[4] R. R. Coifman, P.-L. Lions, Y. Meyer, and S. Semmes, Compensated compactness and Hardy spaces, J. Math. Pures et Appl. 72 (1993), 247-286. MR 95d:46033

[5] R. R. Coifman and Y. Meyer, Au delà des opérateurs pseudo-différentiels, Astérisque 57, 1978. MR 81b:47061

[6] R. R. Coifman and Y. Meyer Wavelets: Calderón-Zygmund and multilinear operators Cambridge Univ. Press, Cambridge, United Kingdom, 1997. MR 98e:42001

[7] G. David and J-L. Journé, A boundedness criterion for generalized Calderón-Zygmund operators, Ann. of Math. 120 (1984), 371-397. MR 85k:42041

[8] G. David, J-L. Journé, and S. Semmes, Opérateurs de Calderón-Zygmund, fonctions paraaccrétives et interpolation, Rev. Mat. Iber. 1 (1985), 1-56. MR 88f:47024

[9] C. Fefferman and E. M. Stein, Some maximal inequalities, Amer. J. Math. 93 (1971), 107-115. MR 44:2026

[10] M. Frazier, B. Jawerth, A discrete transform and decompositions of distribution spaces, J. Func. Anal. 93 (1990), 34-169. MR 92a:46042

[11] M. Frazier, B. Jawerth, and G. Weiss, Littlewood-Paley theory and the study of function spaces, CBMS Regional Conference Series in Mathematics 79, 1991. MR 92m:42021

[12] J. Gilbert and A. Nahmod, Hardy spaces and a Walsh model for bilinear cone operators, Trans. Amer. Math. Soc. 351 (1999), 3267-3300. MR 99m:42022

[13] L. Grafakos and R. Torres, Pseudodifferential operators with homogeneous symbols, Michigan Math. J. 46 (1999), 261-269. MR 2000h:35169

[14] L. Grafakos and R. Torres, A multilinear Schur test and multiplier operators, to appear in J. Func. Anal.

[15] L. Hörmander, Pseudodifferential operators of type 1,1, Comm. Partial Diff. Eq. 13 (1988), 1085-1111. MR 89k:35260

[16] L. Hörmander, Continuity of pseudodifferential operators of type 1,1, Comm. Partial Diff. Eq. 14 (1989), 231-243. MR 90a:35241

[17] M. Lacey and C. Thiele, $L^{p}$ estimates on the bilinear Hilbert transform for $2<p<\infty$, Ann. of Math. 146 (1997), 693-724. MR 99b:42014

[18] Y. Meyer, Regularité des solutions des équations aux dérivées partielles non linéaires, Springer-Verlag Lectures Notes in Math. 842 (1981), 293-302. MR 84c:35015

[19] Y. Meyer, Ondelettes et opérateurs I and II, Hermann, Paris, 1990. MR 93i:42002 MR 93i: 42003

[20] E. M. Stein, Harmonic analysis: Real variable methods, orthogonality, and oscillatory integrals, Princeton University Press, Princeton NJ, 1993. MR 95c:42002

[21] R. Torres, Boundedness results for operators with singular kernels on distribution spaces, Mem. Amer. Math. Soc. 442, 1991. MR 91g:47044

[22] H. Triebel, Theory of function spaces, Monographs in Mathematics, Vol. 78, Birkhauser Verlag, Basel, 1983. MR 86j:46026

[23] A. Youssfi, Bilinear operators and the Jacobian-determinant on Besov spaces, Indiana Univ. Math. J. 45 (1996), 381-396. MR 97h:46050

Department of Mathematics, University of Missouri, Columbia, Missouri 65211

E-mail address: loukas@math.missouri.edu

Department of Mathematics, University of Kansas, Lawrence, Kansas 66049

E-mail address: torres@math.ukans.edu 